\title{
On Mass Spectrum in SQCD, and Problems with the Seiberg Duality. Equal quark masses
}

\author{
Victor L. Chernyak \\ (e-mail: v.l.chernyak@inp.nsk.su) \\ Budker Institute of Nuclear Physics, 630090 Novosibirsk-90, Russia
}

\begin{abstract}
The dynamical scenario is considered for $\mathcal{N}=1 \mathrm{SQCD}$, with $N_{c}$ colors and $N_{c}<N_{F}<$ $3 N_{c}$ flavors with small but nonzero current quark masses $m_{Q} \neq 0$, in which quarks form the diquark-condensate phase. This means that colorless chiral quark pairs condense coherently in the vacuum, $\langle\bar{Q} Q\rangle \neq 0$, while quarks alone don't condense, $\langle Q\rangle=\langle\bar{Q}\rangle=0$, so that the color is confined. Such condensation of quarks results in formation of dynamical constituent masses $\mu_{C} \gg m_{Q}$ of quarks and appearance of light "pions" (similarly to QCD). The mass spectrum of SQCD in this phase is described and comparison with the Seiberg dual description is performed. It is shown that the direct and dual theories are different (except, possibly, for the perturbative strictly superconformal regime).
\end{abstract}

\section{Introduction}

Because supersymmetric gauge theories are much more constrained in comparison with ordinary ones, it is easier for theory to deal with them. So, they can serve, at least, as useful models for elucidating the complicated strong coupling gauge dynamics (not even speaking about their potential relevance to a real world).

The closest to QCD is its supersymmetric extension $\mathcal{N}=1$ SQCD, and it was considered in many papers. We will be dealing here with SQCD in the non-perturbative region (or in the perturbative strong coupling regime). Most impressive results here were obtained by N. Seiberg, who proposed description of this strongly coupled (and/or non-perturbative) SQCD through the equivalent, but weakly coupled dual theory [1] (for reviews, see $[2,3,4]$.

Our purpose in this paper is to introduce in section 3 the main dynamical assumption about the coherent diquark-condensate (DC) phase of SQCD, to describe its consequences for the behavior in the infrared region, the mass spectrum, etc., and to compare with predictions of the Seiberg dual theory. 
The paper is organized as follows. Sections 2 and 4 recall definitions of the direct and dual theories, and some particular examples are considered in section 2. Both direct and dual theories are considered in the conformal window $3 N_{c} / 2<N_{F}<3 N_{c}$ in sections 3 and 5-6, respectively, and at $N_{c}<N_{F}<3 N_{c} / 2$ in section 7 . For completeness, the case $N_{F}>3 N_{c}$ is considered in section 8. Finally, some conclusions are presented in section 9 (and there is one appendix about 't Hooft triangles).

\section{Direct theory . Definition and some examples.}

The fundamental Lagrangian of SQCD with $N_{c}$ colors and $N_{F}$ flavors (at high scale $\left.\mu \gg \Lambda_{Q}\right)$ is given by:

$$
\begin{gathered}
L=\int d^{2} \theta d^{2} \bar{\theta} \operatorname{Tr}\left(Q^{\dagger} e^{V} Q+\bar{Q}^{\dagger} e^{-V} \bar{Q}\right)+ \\
+\int d^{2} \theta\left\{-\frac{2 \pi}{\alpha(\mu)} S+m_{Q}(\mu) \operatorname{Tr} Q \bar{Q}\right\}+\text { h.c. }, \quad \mathrm{S}=\mathrm{W}_{\alpha}^{2} / 32 \pi^{2},
\end{gathered}
$$

where $\alpha(\mu)$ is the running gauge coupling (with its scale parameter $\Lambda_{Q}$, independent of quark masses), $m_{Q}(\mu)$ is the running current quark mass, $W_{\alpha}$ is the gluon field strength, and traces are over color and flavor indices. This theory has the exact $S U\left(N_{c}\right)$ gauge and, in the chiral limit $m_{Q} \rightarrow 0$, global symmetries:

$$
S U\left(N_{F}\right)_{L} \times S U\left(N_{F}\right)_{R} \times U(1)_{B} \times U(1)_{R} .
$$

Under these symmetries, the quarks $Q$ and $\bar{Q}$ transform as:

$$
\begin{gathered}
Q: \quad\left(N_{c}\right)_{\mathrm{col}} \times\left(N_{F}\right)_{L}^{\mathrm{fl}} \times(0)_{R}^{\mathrm{fl}} \times(1)_{B} \times\left(N_{F}-N_{c} / N_{F}\right)_{R}, \\
\bar{Q}: \quad\left(\bar{N}_{c}\right)_{\mathrm{col}} \times(0)_{L}^{\mathrm{fl}} \times\left(\bar{N}_{F}\right)_{R}^{\mathrm{fl}} \times(-1)_{B} \times\left(N_{F}-N_{c} / N_{F}\right)_{R} .
\end{gathered}
$$

The explicit dependence of the gluino condensate $\langle S\rangle$ on the current quark masses and $\Lambda_{Q}$ can be found as follows.

a) One can start with $N_{F}<3 N_{c}$ and the heavy quarks, $m_{Q}^{\text {pole }} \equiv m_{Q}\left(\mu=m_{Q}^{\text {pole }}\right) \gg \Lambda_{Q}$, so that the theory is UV-free and in the weak coupling regime at sufficiently large $\mu$.

b) Then, to integrate out all quarks directly in the perturbation theory at scales $\mu<\mu_{H}=m_{Q}^{\text {pole }}$, resulting in the pure Yang-Mills theory with the scale factor $\Lambda_{Y M}$. The value of $\Lambda_{\mathrm{YM}}$ can be found from the matching of couplings $\alpha_{+}(\mu)$ and $\alpha_{-}(\mu)$ of the upper and lower theories at $\mu=\mu_{H}: \alpha_{+}\left(\mu_{H}\right)=\alpha_{-}\left(\mu_{H}\right)$. The upper theory is always the original one with $N_{c}$ colors and $N_{F}$ flavors, and the value of $\alpha_{+}\left(\mu_{H}\right)$ can be obtained starting with high $\mu \gg \mu_{H}$ and evolving down to $\mu=\mu_{H}$ through the standard perturbative RG-flow for theory with $N_{c}$ colors and $N_{F}$ flavors of massless quarks. 1 But instead, the same

\footnotetext{
1 In (2.2),(2.3) and everywhere below in the text the perturbative NSVZ [5] $\beta$-function is used, corresponding to the Pauli-Villars scheme.
} 
value $\alpha_{+}(\mu)$ can be obtained starting with $\mu \sim \Lambda_{Q}$ and going up to $\mu=\mu_{H}$ with the same RG-flow for massless quarks. I.e. $\left(\mathrm{g}^{2}(\mu)=4 \pi \alpha(\mu), \mathrm{b}_{\mathrm{o}}=3 \mathrm{~N}_{\mathrm{c}}-\mathrm{N}_{\mathrm{F}}\right)$ :

$$
\frac{2 \pi}{\alpha_{+}\left(\mu_{H}\right)}=\mathrm{b}_{\mathrm{o}} \ln \left(\frac{\mu_{\mathrm{H}}}{\Lambda_{\mathrm{Q}}}\right)-\mathrm{N}_{\mathrm{F}} \ln \left(\frac{1}{z_{\mathrm{Q}}\left(\mu_{\mathrm{H}}, \Lambda_{\mathrm{Q}}\right)}\right)+\mathrm{N}_{\mathrm{c}} \ln \left(\frac{1}{\mathrm{~g}^{2}\left(\mu_{\mathrm{H}}\right)}\right)+\mathrm{C}_{+},
$$

where $z_{Q}=z_{Q}\left(\mu_{H}, \Lambda_{Q}\right) \ll 1$ is the standard perturbative renormalization factor (logarithmic in this case) of massless quarks in theory with $N_{c}$ colors and $N_{F}$ flavors.

As for the lower theory, in all examples considered in this section it is the Yang-Mills one with $N_{c}^{\prime}$ colors and no quarks. Its coupling can be written in a similar way as:

$$
\frac{2 \pi}{\alpha_{-}\left(\mu_{H}\right)}=3 N_{c}^{\prime} \ln \left(\frac{\mu_{H}}{\Lambda_{\mathrm{YM}}}\right)+N_{c}^{\prime} \ln \left(\frac{1}{\mathrm{~g}^{2}\left(\mu_{H}\right)}\right)+C_{-} .
$$

$C_{ \pm}$in (2.2),(2.3) are constants independent of the quark mass values. Our purpose here and everywhere below is to trace explicitly the dependence on the parameters like $\mu_{H} / \Lambda_{Q}$ which will be finally expressed through the universal parameter $m_{Q} / \Lambda_{Q}, m_{Q} \equiv m_{Q}(\mu=$ $\Lambda_{Q}$ ), which can be large $m_{Q} / \Lambda_{Q} \gg 1$, or small $m_{Q} / \Lambda_{Q} \ll 1$. So, from now on and everywhere below the constant terms like $C_{ \pm}$will be omitted, as their effect is equivalent to a redefinition of $\Lambda_{Q}$ by a constant factor. ${ }^{2}$

In the case considered now : $N_{c}^{\prime}=N_{c}, \mu_{H}=m_{Q}^{\text {pole }} \gg \Lambda_{Q}$, and one obtains then from $(2.2),(2.3)$ :

$$
\Lambda_{Y M}=\left(\Lambda_{Q}^{\mathrm{b}_{\mathrm{o}}} \operatorname{det} m_{Q}\right)^{1 / 3 N_{c}}, \quad m_{Q} \equiv z_{Q}^{-1}\left(m_{Q}^{\text {pole }}, \Lambda_{Q}\right) m_{Q}^{\text {pole }} \gg m_{Q}^{\text {pole }} \gg \Lambda_{Q} .
$$

c) Lowering the scale $\mu$ down to $\mu<\Lambda_{Y M}$ and integrating out all gauge degrees of freedom, except for the one whole field $S$ itself, one can write the effective Lagrangian in the Veneziano-Yankielowicz (VY) form [6], from which one obtains the gluino condensate:

$$
\langle S\rangle=\Lambda_{Y M}^{3}=\left(\Lambda_{Q}^{\mathrm{b}_{\mathrm{o}}} \operatorname{det} m_{Q}\right)^{1 / N_{c}}, \quad m_{Q}=m_{Q}\left(\mu=\Lambda_{Q}\right) .
$$

Now, the expression (2.5) can be continued in $m_{Q}$ from large $m_{Q} \gg \Lambda_{Q}$ to small values, $m_{Q} \ll \Lambda_{Q}$. While $m_{Q}$ for $m_{Q} \gg \Lambda_{Q}$ is some formally defined parameter (see (2.4), the physical quark mass is $m_{Q}^{\text {pole }} \gg \Lambda_{Q}$ and it does not run any more at $\mu<m_{Q}^{\text {pole }}$ ), at $m_{Q} \ll \Lambda_{Q}$ it has a simple and direct meaning: $m_{Q}=m_{Q}\left(\mu=\Lambda_{Q}\right)$.

The expression (2.5) for $\langle S\rangle$ appeared in the literature many times before, but to our knowledge, the exact definition of the parameter $m_{Q}$ entering (2.5), i.e. its relation with $m_{Q}(\mu)$ entering $(1)$ which defines the theory, has not been given. Clearly, without this explicit relation the expression (2.5) has no much meaning, as the quark mass parameter $m_{Q}(\mu)$ is running. For instance, if $m_{Q}$ is understood as $m_{Q}^{\text {pole }}$ in (2.5) for heavy quarks, the relation $\langle S\rangle=\left(\Lambda_{Q}^{\mathrm{bo}} \operatorname{det} m_{Q}^{\text {pole }}\right)^{1 / N_{c}}$ will be erroneous. All this becomes especially important, in particular, at $3 N_{c} / 2<N_{F}<3 N_{c}$ and $m_{Q} \ll \Lambda_{Q}$, when $m_{Q}(\mu)$ runs in a power-like fashion: $m_{Q}\left(\mu_{2}\right)=\left(\mu_{1} / \mu_{2}\right)^{\mathrm{b}_{\circ} / \mathrm{N}_{\mathrm{F}}} m_{Q}\left(\mu_{1}\right)$. Everywhere below, except for the section 8 , only the case $m_{Q} \ll \Lambda_{Q}$ will be considered.

2 Introducing the Wilsonian coupling $\alpha_{W}(\mu)$ whose $\beta$-function is that of NSVZ for $\alpha(\mu)$ but without the denominator, $2 \pi / \alpha_{W}(\mu)=2 \pi / \alpha(\mu)-N_{c} \ln \left(1 / \mathrm{g}^{2}(\mu)\right)$ [5], one has: $C_{+}=2 \pi / \alpha_{W}^{+}\left(\mu=\Lambda_{Q}\right), C_{-}=$ $2 \pi / \alpha_{W}^{-}\left(\mu=\Lambda_{Y M}\right)$. In essence, the term $N_{c} \ln \left(1 / \mathrm{g}^{2}\left(\mu_{H}\right)\right)$ in $(2.2)$ is the higher loop perturbative renormalization factor of gluons, i.e. $N_{c} \ln \left(z_{\mathrm{g}}\left(\mu_{H}, \Lambda_{Q}\right)\right)=N_{c} \ln \left(\alpha_{+}\left(\mu_{H}\right) / \alpha_{+}\left(\mu=\Lambda_{Q}\right)\right)$, and similarly in (2.3). 
d) From the Konishi anomaly equation [7]:

$$
\left\langle\left(\bar{Q}_{\bar{j}} Q^{i}\right)_{\mu}\right\rangle=\left(m_{Q}^{-1}(\mu)\right)_{\bar{j}}^{i}\langle S\rangle
$$

one obtains the explicit value of the chiral condensate:

$$
\begin{gathered}
\left\langle\left(\bar{Q}_{\bar{j}} Q^{i}\right)_{\mu=\Lambda_{Q}}\right\rangle \equiv \mathcal{M}_{\mathrm{ch}}^{2} \delta \frac{i}{j}=\frac{\langle S\rangle}{m_{Q}} \delta \frac{i}{j}, \quad \mathcal{M}_{\mathrm{ch}}=\left(\Lambda_{Q}^{\mathrm{b}_{\mathrm{o}}} m_{Q}^{\bar{N}_{c}}\right)^{1 / 2 N_{c}}, \quad \bar{N}_{c}=N_{F}-N_{c}, \\
\langle S\rangle=\Lambda_{Y M}^{3}=\left(\Lambda_{Q}^{\mathrm{b}_{\mathrm{o}}} \operatorname{det} m_{Q}\right)^{1 / N_{c}}, \quad m_{Q} \ll \Lambda_{Q} .
\end{gathered}
$$

Now, the expression (2.5) can be continued in $N_{F}$ from the region $N_{F}<3 N_{c}$ to $N_{F}>3 N_{c}$ and, together with the Konishi anomaly relation (2.6), these two become then the basic universal relations for any values of quark masses and any $N_{F}$.

To check this universal form of (2.5), let us consider briefly (see section 8 for more detail) the case $N_{F}>3 N_{c}$ and $m_{Q} \ll \Lambda_{Q}$. In this case $\mathrm{b}_{\mathrm{o}}=\left(3 \mathrm{~N}_{\mathrm{c}}-\mathrm{N}_{\mathrm{F}}\right)<0$, so that the theory is IR-free in the interval $\mu_{H}<\mu<\Lambda_{Q}$, where $\mu_{H}$ is the highest physical mass $\left(\Lambda_{Y M} \ll \mu_{H}=m_{Q}^{\text {pole }} \ll \Lambda_{Q}\right.$ in this example). I.e., its coupling which is $O(1)$ at $\mu=\Lambda_{Q}$ becomes logarithmically small at $\mu \ll \Lambda_{Q}$. Besides, the parameter $m_{Q}$ has now a direct physical meaning as the value of the running quark mass at $\mu=\Lambda_{Q}, m_{Q} \equiv m_{Q}\left(\mu=\Lambda_{Q}\right) \ll \Lambda_{Q}$. So, starting with $\mu=\Lambda_{Q}$ and going down perturbatively to $\mu_{H}=m_{Q}^{\text {pole }}=m_{Q}\left(\mu=m_{Q}^{\text {pole }}\right)=z_{Q}^{-1}\left(\Lambda_{Q}, m_{Q}^{\text {pole }}\right) m_{Q} \gg m_{Q}$ $\left(z_{Q}\left(\Lambda_{Q}, m_{Q}^{\text {pole }}\right) \ll 1\right.$ is the perturbative logarithmic renormalization factor of massless quarks), one can integrate then out all quarks as heavy ones. Writing the matching condition for two couplings $\alpha_{+}$and $\alpha_{-}$, one obtains (2.2),(2.3) with the only replacement: $z_{Q}\left(m_{Q}^{\text {pole }} \gg \Lambda_{Q}, \Lambda_{Q}\right) \rightarrow z_{Q}^{-1}\left(\Lambda_{Q}, m_{Q}^{\text {pole }} \ll \Lambda_{Q}\right)$, and the same expression (2.5).

Another check can be performed for $N_{F}<N_{c}-1$ and small quark masses, $m_{Q} \ll \Lambda_{Q}$. In this case all quarks are higgsed and the gauge symmetry $S U\left(N_{c}\right)$ is broken down to $S U\left(N_{c}^{\prime}=N_{c}-N_{F}\right)$ at the high scale $\mu_{H}=\mu_{\mathrm{gl}} \gg \Lambda_{Q}:\left\langle Q_{a}^{i}\right\rangle_{\mu=\mu_{\mathrm{gl}}}=\delta_{a}^{i} \mathcal{M}_{o},\left\langle\bar{Q}_{\bar{j}}^{a}\right\rangle_{\mu=\mu_{\mathrm{gl}}}=$ $\delta_{\bar{j}} \mathcal{M}_{o}, \mathcal{M}_{o} \gg \Lambda_{Q}$. $\left(2 N_{c} N_{F}-N_{F}^{2}\right)$ gluons become massive, with the mass scale $\mu_{\mathrm{gl}}^{2}=$ $\mathrm{g}_{+}^{2}\langle\hat{\Pi}\rangle=\mathrm{g}_{+}^{2} \mathcal{M}_{o}^{2}, \mathrm{~g}_{+}^{2}=4 \pi \alpha_{+}\left(\mu=\mu_{\mathrm{gl}}, \Lambda_{Q}\right) \ll 1$. The same number of quark degrees of freedom acquire the same masses and become the superpartners of massive gluons (in a sense, they can be considered as the heavy "constituent quarks"), and there remain $N_{F}^{2}$ light complex pion fields $\hat{\pi}_{\bar{j}}^{i}: \hat{\Pi}_{\bar{j}}^{i}=\left(\bar{Q}_{\bar{j}} Q^{i}\right)_{\mu=\mu_{\mathrm{gl}}}=\mathcal{M}_{o}^{2}\left(\delta \frac{i}{j}+\hat{\pi}_{\bar{j}}^{i} / \mathcal{M}_{o}\right),\left\langle\hat{\Pi}_{\bar{j}}^{i}\right\rangle=\delta_{\bar{j}}^{i} \mathcal{M}_{o}^{2}$.

All heavy particles can be integrated out at scales $\mu<\mu_{\mathrm{gl}}$. The numerical matching of couplings at $\mu_{H}=\mu_{\mathrm{gl}}: \alpha_{+}\left(\mu=\mu_{\mathrm{gl}}, \Lambda_{Q}\right)$ in (2.2), i.e. those of the original theory, with $\mu_{\mathrm{gl}}^{2}=\mathrm{g}_{+}^{2}\langle\hat{\Pi}\rangle=\mathrm{g}_{+}^{2} \mathcal{M}_{o}^{2}, \hat{\Pi}=(\bar{Q} Q)_{\mu=\mu_{\mathrm{gl}}}$, and $\alpha_{-}\left(\mu=\mu_{\mathrm{gl}}, \Lambda_{L}\right)$ in $(2.3)$ of the lower energy pure Yang-Mills theory can be performed similarly to the previous examples with heavy quarks. But in this case we consider it will be more useful to write the explicit form of the $\hat{\Pi}$-dependence of the lower energy coupling $\alpha_{-}\left(\mu<\mu_{\mathrm{gl}}, \Lambda_{L}\right)$ multiplying the field strength squared of massless gluons, to see how the multi-loop $\beta$ - function reconciles with the holomorphic dependence of $\Lambda_{L}$ on the chiral superfields $\hat{\Pi}$. This looks now as :

$$
\frac{2 \pi}{\alpha_{-}\left(\mu<\mu_{\mathrm{gl}}, \Lambda_{L}\right)}=\left\{3\left(N_{c}-N_{F}\right) \ln \left(\frac{\mu}{\Lambda_{Q}}\right)+\left(N_{c}-N_{F}\right) \ln \left(\frac{1}{\mathrm{~g}_{-}^{2}\left(\mu,\left\langle\Lambda_{L}\right\rangle\right)}\right)\right\}+
$$




$$
\begin{aligned}
& +\left\{\frac{3}{2} \ln \left(\frac{\mathrm{g}_{+}^{2 N_{F}}\left(\mu=\mu_{\mathrm{gl}}, \Lambda_{Q}\right) \operatorname{det} \hat{\Pi}}{\Lambda_{Q}^{2 N_{F}}}\right)+N_{F} \ln \left(\frac{1}{\mathrm{~g}_{+}^{2}\left(\mu=\mu_{\mathrm{gl}}, \Lambda_{Q}\right)}\right)\right\}- \\
& -\left\{\frac{1}{2} \ln \left(\frac{\mathrm{g}_{+}^{2 N_{F}}\left(\mu=\mu_{\mathrm{gl}}, \Lambda_{Q}\right) \operatorname{det} \hat{\Pi}}{\Lambda_{Q}^{2 N_{F}}}\right)+N_{F} \ln \left(\frac{1}{z_{Q}\left(\mu=\mu_{\mathrm{gl}}, \Lambda_{Q}\right)}\right)\right\},
\end{aligned}
$$

where three terms in curly brackets in (2.8) are the contributions of, respectively, massless gluons, massive gluons, and higgsed quarks.

It is worth noting that the dependence of the coupling $2 \pi / \alpha_{-}$on the quantum pion superfields $\hat{\pi}_{\frac{i}{j}}^{i} / \mathcal{M}_{o}$ entering $\hat{\Pi}_{j}^{i}$ originates only from the $\hat{\pi} / \mathcal{M}_{o}$-dependence of heavy particle masses entering the "normal" one-loop contributions to the gluon vacuum polarization, while the "anomalous" higher loop contributions [5] originating from the quark and gluon renormalization factors $z_{Q}$ and $z_{\mathrm{g}}^{ \pm} \sim \mathrm{g}_{ \pm}^{2}$ (see the footnote 2) do not contain the quantum pion fields $\hat{\pi} / \mathcal{M}_{o}$ and enter (2.8) as pure neutral c-numbers. This is clear from the R-charge conservation (see the footnote 3 ) or, equivalently, from the holomorphic dependence of F-terms on chiral quantum superfields (the chiral superfields here are $\left.\bar{Q} \bar{j} Q^{i}\left(\mu_{1}\right)=z_{Q}\left(\mu_{1}, \mu_{2}\right) \bar{Q} \bar{j}^{i}\left(\mu_{2}\right)\right)$.

So, the coupling $\alpha_{-}\left(\mu, \Lambda_{L}\right)$ of the lower energy pure Yang-Mills theory at $\mu<\mu_{\mathrm{gl}}$ and its scale factor $\Lambda_{L}$ look as:

$$
\begin{gathered}
\frac{2 \pi}{\alpha_{-}^{W}\left(\mu<\mu_{\mathrm{gl}}, \Lambda_{L}\right)}=\frac{2 \pi}{\alpha_{-}\left(\mu<\mu_{\mathrm{gl}}, \Lambda_{L}\right)}-\left(N_{c}-N_{F}\right) \ln \frac{1}{\mathrm{~g}_{-}^{2}\left(\mu<\mu_{\mathrm{gl}},\left\langle\Lambda_{L}\right\rangle\right)}=3\left(N_{c}-N_{F}\right) \ln \left(\frac{\mu}{\Lambda_{L}}\right) \\
\Lambda_{L}^{3\left(N_{c}-N_{F}\right)}=\frac{\Lambda_{Q}^{\mathrm{b}_{\mathrm{o}}}}{z_{Q}^{N_{F}}\left(\mu_{\mathrm{gl}}, \Lambda_{Q}\right) \operatorname{det} \hat{\Pi}} \equiv \frac{\Lambda_{Q}^{\mathrm{b}_{\mathrm{o}}}}{\operatorname{det} \Pi}=\Lambda_{Y M}^{3\left(N_{c}-N_{F}\right)}\left(\operatorname{det} \frac{\langle\Pi\rangle}{\Pi}\right) \\
\Pi \equiv z_{Q}\left(\mu_{\mathrm{gl}}, \Lambda_{Q}\right) \hat{\Pi}, \quad\langle\Pi\rangle=\mathcal{M}_{\mathrm{ch}}^{2} \ll \mathcal{M}_{o}^{2}, \quad\left\langle\Lambda_{L}\right\rangle=\Lambda_{Y M}=\left(\Lambda_{Q}^{\mathrm{b}_{\mathrm{o}}} \operatorname{det} m_{Q}\right)^{1 / 3 N_{c}},(2.10)
\end{gathered}
$$

and the Lagrangian at $\mu<\mu_{\mathrm{gl}}$ takes the form : 3

$$
L=\int d^{2} \theta d^{2} \bar{\theta}\left\{2 \operatorname{Tr} \sqrt{\hat{\Pi}^{\dagger} \hat{\Pi}}\right\}+\int \mathrm{d}^{2} \theta\left\{-\frac{2 \pi}{\alpha_{-}\left(\mu, \Lambda_{\mathrm{L}}\right)} \hat{\mathrm{S}}+\hat{\mathrm{m}}_{\mathrm{Q}} \operatorname{Tr} \hat{\Pi}\right\},
$$

where $\hat{S}=\hat{W}_{\alpha}^{2} / 32 \pi^{2}$, and $\hat{W}_{\alpha}$ are the gauge field strengths of $\left(N_{c}-N_{F}\right)^{2}-1$ remaining massless gluon fields.

Lowering the scale $\mu$ down to $\mu<\Lambda_{Y M}$ and integrating out all gauge degrees of freedom, except for the one whole field $\hat{S}$ itself (this leaves behind a large number of gluonia with masses $M_{\mathrm{gl}} \sim \Lambda_{Y M}$ ), one obtains the VY - form :

$$
L=\int d^{2} \theta d^{2} \bar{\theta}\left\{2 \operatorname{Tr} \sqrt{\hat{\Pi}^{\dagger} \hat{\Pi}}+(\text { D terms of the field } \hat{\mathrm{S}})\right\}+
$$

3 Because the gluon fields are not yet integrated completely, there are the gluon regulator fields (implicit) whose contributions ensure the R-charge conservation in (2.11), see also (2.12) below.

Besides, we neglected in (2.11) the additional dependence of the Kahler term on the quantum pion fields $\hat{\pi} / \mathcal{M}_{o}$ (originating from the dependence on $\hat{\pi} / \mathcal{M}_{o}$ of the quark renormalization factor $z_{Q}\left(\hat{\Pi}^{\dagger}, \hat{\Pi}\right)$ ), because at the weak coupling this will influence the pion mass values through logarithmically small corrections only. 


$$
+\int d^{2} \theta\left\{-\left(N_{c}-N_{F}\right) \hat{S}\left(\ln \left(\frac{\hat{S}}{\Lambda_{L}^{3}}\right)-1\right)+\hat{m}_{Q} \operatorname{Tr} \hat{\Pi}\right\}, \mu \lesssim \Lambda_{\mathrm{YM}}
$$

It is worth noting that it is the first place where the non-perturbative effects were incorporated to obtain the VY - form of the superpotential (the non-perturbative effects introduce the infrared cutoff $\sim \Lambda_{Y M}$, so that the explicit dependence on $\mu$ disappears at $\mu<\Lambda_{Y M}$ ), while all previous calculations with this example were purely perturbative. One obtains from (2.12) the gluino vacuum condensate: $\langle\hat{S}\rangle=\left\langle\Lambda_{L}^{3}\right\rangle=\Lambda_{Y M}^{3}=\langle S\rangle=\left(\Lambda_{Q}^{\mathrm{b}_{\mathrm{o}}} \operatorname{det} m_{Q}\right)^{1 / N_{c}}$.

Finally, integrating out the last gluonium field $\hat{S}$ (with its mass scale $\sim \Lambda_{Y M}$ ) at lower energies, one obtains the Lagrangian of pions:

$$
\begin{aligned}
& L=\left[2 \operatorname{Tr} \sqrt{\hat{\Pi}^{\dagger} \hat{\Pi}}\right]_{\mathrm{D}}+\left[\left(\mathrm{N}_{\mathrm{c}}-\mathrm{N}_{\mathrm{F}}\right)\left(\frac{\Lambda_{\mathrm{Q}}^{\mathrm{b}_{\mathrm{o}}}}{z_{\mathrm{Q}}\left(\mu_{\mathrm{gl}}, \Lambda_{\mathrm{Q}}\right) \operatorname{det} \hat{\Pi}}\right)^{1 /\left(\mathrm{N}_{\mathrm{c}}-\mathrm{N}_{\mathrm{F}}\right)}+\hat{\mathrm{m}}_{\mathrm{Q}} \operatorname{Tr} \hat{\Pi}\right]_{\mathrm{F}}=(2.13) \\
= & {\left[\frac{2}{z_{Q}\left(\mu_{\mathrm{gl}}, \Lambda_{Q}\right)} \operatorname{Tr} \sqrt{\Pi^{\dagger} \Pi}\right]_{\mathrm{D}}+\left[\left(\mathrm{N}_{\mathrm{c}}-\mathrm{N}_{\mathrm{F}}\right)\left(\frac{\Lambda_{\mathrm{Q}}^{\mathrm{b}_{\mathrm{o}}}}{\operatorname{det} \Pi}\right)^{1 /\left(\mathrm{N}_{\mathrm{c}}-\mathrm{N}_{\mathrm{F}}\right)}+\mathrm{m}_{\mathrm{Q}} \operatorname{Tr} \Pi\right]_{\mathrm{F}}, \quad \mu \ll \Lambda_{\mathrm{YM}} . }
\end{aligned}
$$

The superpotential of the form $\left(N_{c}-N_{F}\right)\left(\Lambda_{Q}^{\mathrm{b}_{\mathrm{o}}} / \operatorname{det} \Pi\right)^{1 / \bar{N}_{c}}$ appeared many times in the literature because, up to an absolute normalization of the field $\Pi$ (which is not RGinvariant by itself), this is the only possible form of the superpotential, if one is able to show that the lowest energy Lagrangian depends on $N_{F}^{2}$ pion superfields only. But it seems, the absolute normalization of all terms entering (2.13) has never been carefully specified (clearly, the absolute normalization makes sense only when both the superpotential and the Kahler terms are absolutely normalized simultaneously). The Lagrangian (2.13) describes weakly interacting pions with small masses $M_{\pi}=2 \hat{m}_{Q}=2 z_{Q}\left(\mu_{\mathrm{gl}}, \Lambda_{Q}\right) m_{Q} \ll$ $m_{Q} \ll \Lambda_{Y M} \ll \Lambda_{Q}$.

On the whole, the mass spectrum contains in this case: $\left(2 N_{c} N_{F}-N_{F}^{2}\right)$ massive gluons and "constituent quarks" with the mass scale $\mu_{\mathrm{gl}}=\mathrm{g}_{H} \mathcal{M}_{o} \gg \Lambda_{Q}$, a large number of gluonia with the mass scale $\sim \Lambda_{Y M} \ll \Lambda_{Q}$, and $N_{F}^{2}$ pions with small masses $M_{\pi}=$ $2 \hat{m}_{Q}=2 m_{Q}\left(\mu=\mu_{\mathrm{gl}}\right) \ll \Lambda_{Y M}$.

The form (2.13) can be continued in $N_{F}$ to the point $N_{F}=N_{c}-1$ and it predicts then the form of the pion Lagrangian for this case. Now, the whole gauge group is higgsed at the high scale $\mu_{H}=\mu_{\mathrm{gl}} \gg \Lambda_{Q}$, and the direct way to obtain (2.13) is not through the VY - procedure, but through the calculation of the one-instanton contribution [8] [11]. The changes in the mass spectrum are evident and, most important, - there is no confinement and there are no particles with masses $\sim \Lambda_{Y M}$ in the spectrum in this case.

\section{Direct theory. Conformal window $3 N_{c} / 2<N_{F}<3 N_{c}$}

The superconformal behavior means the absence of the scale $\Lambda_{Q}$ in the physical mass spectrum. In other words, there are no particles with masses $\sim \Lambda_{Q}$, all quarks and gluons remain effectively massless at $\mu_{H} \ll \mu \ll \Lambda_{Q}$, where $\mu_{H}$ is the highest physical mass scale. So, "nothing especially interesting" happens when decreasing the scale $\mu$ from $\mu \gg \Lambda_{Q}$ down to $\mu_{H} \ll \mu<\Lambda_{Q}$. Only the character of running of the coupling $\alpha(\mu)$ and the 
quark renormalization factor $z_{Q}(\mu)$ change. The slow logarithmic evolution in the weak coupling region $\mu \gg \Lambda_{Q}$ is replaced by freezing of $\alpha(\mu)$ at $\mu<\Lambda_{Q}: \alpha(\mu) \rightarrow \alpha^{*}$, while $z_{Q}(\mu)$ acquires the power behavior: $z_{Q}\left(\Lambda_{Q}, \mu\right)=\left(\mu / \Lambda_{Q}\right)^{\mathrm{b}_{\mathrm{o}} / \mathrm{N}_{\mathrm{F}}}<1$. As a result, the Green functions of chiral superfields also behave in a power-like fashion, with dynamical dimensions determined by their R-charges: $\mathrm{D}=3|\mathrm{R}| / 2$. This conformal regime continues until $\mu$ reaches at $\mu \ll \Lambda_{Q}$ the highest physical mass scale $\mu_{H} \ll \Lambda_{Q}$, and then the conformal behavior breaks down.

There are three characteristic scales at $\mu=\Lambda_{Q}$ in the direct theory: the current quark mass $m_{Q}$, the scale $\mathcal{M}_{\mathrm{ch}}$ of its chiral vacuum condensate, and the scale $\Lambda_{Y M}$ of the gluino condensate. It is seen from (2.5-2.7) that in the whole region $N_{c}<N_{F}<3 N_{c}$ there is an hierarchy:

$$
m_{Q} \ll \Lambda_{Y M} \ll \mathcal{M}_{\text {ch }} \quad \text { at } \quad N_{c}<N_{F}<3 N_{c} .
$$

By itself, this hierarchy has no direct physical consequences, until it is realized that some physical masses stay behind the above quantities. As will be shown below, within the dynamical scenario considered, the above inequalities reflect a real hierarchy of physical masses: $m_{Q}$ is the mass of lightest pions, $\Lambda_{Y M}$ is the mass scale of gluonia and $\mathcal{M}_{\mathrm{ch}}$ is the dynamical constituent mass of quarks.

The main idea of the dynamical scenario for SQCD, with $N_{c}<N_{F}<3 N_{c}$ and small equal quark masses, considered in this paper is that this theory is in the collective coherent "diquark-condensate" (DC) phase. This means that quarks don't condense alone, $\left\langle Q^{i}\right\rangle=$ $\left\langle\bar{Q}_{\bar{j}}\right\rangle=0$ (because there are too many flavors at $N_{f}>N_{c}$ ). In other words, theory is not higgsed by quarks, all gluons remain massless at scales $\mu \gg \Lambda_{Y M}$, and color is confined. But quarks condense in colorless chiral pairs $\left(\bar{Q}_{\bar{j}} Q^{i}\right)$ and these pairs form the coherent condensate ( like the quark-antiquark pairs in the Nambu-Jona-Lasinio model and, more importantly, like QCD). And as a result of this coherent condensation, quarks acquire large (in comparison with their pole mass, $m_{Q}^{\text {pole }}=m_{Q}\left(\mu=m_{Q}^{\text {pole }}\right)$ ) dynamical constituent mass $\mu_{C}^{2}=\left\langle\Pi_{2}\right\rangle=\left\langle(\bar{Q} Q)_{\mu=\mu_{2}}\right\rangle,\left(\mu_{2}=\mu_{C} /(\right.$ several $), \mu_{\mathrm{C}}=\mathcal{M}_{\mathrm{ch}}$, see below). This constituent quark mass $\mu_{C}=\mathcal{M}_{\mathrm{ch}}$ is the highest physical mass $\mu_{H}$ and it stops the massless perturbative RG-evolution at scales $\mu<\mu_{C}$. Simultaneously, the light composite pions $\pi_{\bar{j}}^{i}$ are formed, with masses $M_{\pi} \sim m_{2}=m_{Q}\left(\mu=\mu_{2}\right),\left(m_{2}=m_{Q}\right.$, see below). 4

All this occurs in the "threshold region" $\mu_{2}=\mu_{C} /$ (several) $<\mu<\mu_{1}=$ (several) $\mu_{C}$ around the scale $\mu_{C}$ of the constituent quark mass. In other words, the non-perturbative effects operate in this threshold region, so that they "turn on" at $\mu=\mu_{1}$ and "saturate" at $\mu=\mu_{2}$.

${ }^{4}$ This is unlike (our) QCD, where the value of the constituent quark mass $\mu_{C}$ is also determined by the coherent chiral quark condensate, $\mu_{C}^{3}=\langle\psi \bar{\psi}\rangle$, but it is here $\mu_{C} \sim \Lambda_{Q}$, while $m_{\pi} \sim\left(m_{Q} \mu_{C}\right)^{1 / 2}$. The difference in parametrical dependence of $m_{\pi}$ on the current quark mass $m_{Q}$ between SQCD and QCD is because the spin 1/2 quarks are condensed in QCD, while these are spin zero quarks in SQCD.

Besides, unlike the genuine spontaneous breaking of the chiral flavor symmetry in QCD with $\mu_{C}=$ $\langle\psi \bar{\psi}\rangle^{1 / 3} \sim \Lambda_{Q} \neq 0$ at $m_{Q} \rightarrow 0$, in SQCD $\mu_{C}=\left\langle\bar{Q} Q_{\mu=\Lambda_{Q}}\right\rangle^{1 / 2}=\mathcal{M}_{\text {ch }} \rightarrow 0$ at $m_{Q} \rightarrow 0$, see (2.7). Nevertheless, because the ratio $\mathcal{M}_{\mathrm{ch}} / m_{Q} \gg 1$ is parametrically large at $m_{Q} \ll \Lambda_{Q}$, all qualitative features remain the same, so that this can be considered as the "quasi-spontaneous breaking" of the chiral flavor symmetry. 
If this idea is accepted, the proposed effective Lagrangian at the scale $\mu_{2}$ has the form:

$$
\begin{gathered}
L=\int d^{2} \theta d^{2} \bar{\theta}\left\{\operatorname{Tr} \sqrt{\Pi_{2}^{\dagger} \Pi_{2}}+\mathrm{Z}_{2} \operatorname{Tr}\left(\mathrm{Q}_{2}^{\dagger} \mathrm{e}^{\mathrm{V}} \mathrm{Q}_{2}+\overline{\mathrm{Q}}_{2}^{\dagger} \mathrm{e}^{-\mathrm{v}} \overline{\mathrm{Q}}_{2}\right)+\cdots\right\}+ \\
+\int d^{2} \theta\left(\mathrm{W}_{\mathrm{g}}+W_{Q}\right)+\text { h.c., } \quad \mathrm{W}_{\mathrm{g}}=-\frac{2 \pi}{\alpha\left(\mu_{2}\right)} \mathrm{S}, \quad \mathrm{S}=\mathrm{W}_{\alpha}^{2} / 32 \pi^{2}, \\
W_{Q}=\left(\frac{\operatorname{det} \Pi_{2}}{\Lambda_{Q}^{\mathrm{b}}}\right)^{1 / \bar{N}_{c}} \operatorname{Tr}\left(\overline{\mathrm{Q}}_{2} \Pi_{2}^{-1} \mathrm{Q}_{2}\right)-\mathrm{N}_{\mathrm{F}}\left(\frac{\operatorname{det} \Pi_{2}}{\Lambda_{\mathrm{Q}}^{\mathrm{b}}}\right)^{1 / \overline{\mathrm{N}}_{\mathrm{c}}}+\mathrm{m}_{2} \operatorname{Tr} \Pi_{2}, \\
Z_{2}=\frac{\Lambda_{o}}{\mu_{C}}=\left(\frac{\mu_{C}}{\Lambda_{Q}}\right)^{\mathrm{b}_{\mathrm{o}} / \overline{\mathrm{N}}_{\mathrm{c}}}=\frac{m_{Q}}{\mathcal{M}_{\mathrm{ch}}}, \quad \Lambda_{o}=\frac{1}{\left\langle\Pi_{2}\right\rangle}\left(\frac{\operatorname{det}\left\langle\Pi_{2}\right\rangle}{\Lambda_{Q}^{\mathrm{b}_{\mathrm{o}}}}\right)^{1 / \bar{N}_{c}}, \quad \bar{N}_{c}=N_{F}-N_{c} .
\end{gathered}
$$

Here, the field $\left(\Pi_{2}\right)_{\bar{j}}^{i}=\left(\bar{Q}_{\bar{j}} Q^{i}\right)_{\mu=\mu_{2}}^{\text {(light) }}$ represents the dynamically generated "oneparticle light part" of the composite field: $\left(\Pi_{2}\right) \frac{i}{j}=\mu_{C}^{2}\left(\delta \frac{i}{j}+\pi_{j}^{i} / \mu_{C}\right)$, it contains the c-number vacuum part $\mu_{C}^{2} \delta_{\bar{j}}^{i}=\left\langle\left(\Pi_{2}\right) \frac{i}{j}\right\rangle=\left\langle\bar{Q}_{2, \bar{j}} Q_{2}^{i}\right\rangle \equiv\left\langle\bar{Q}_{\bar{j}} Q^{i}\right\rangle_{\mu=\mu_{2}}$, and the quantum fields $\pi_{\bar{j}} / \mu_{C}$ of light pions. The canonically normalized quark fields $C_{2}=Z_{2}^{1 / 2} Q_{2}$ and $\bar{C}_{2}=Z_{2}^{1 / 2} \bar{Q}_{2}$ have no c-number vacuum parts, $\langle C\rangle=\langle\bar{C}\rangle=0$, and are the quantum fields of heavy constituent quarks with "the field masses" $\left(\mu_{C}\right)_{i}^{\bar{j}}$ and c-number masses $\mu_{C}$ :

$$
\left(\mu_{C}\right)_{i}^{\bar{j}}=\frac{1}{Z_{2}}\left(\frac{\operatorname{det} \Pi_{2}}{\Lambda_{Q}^{b_{o}}}\right)^{1 / \bar{N}_{c}}\left(\Pi_{2}^{-1}\right)_{i}^{\bar{j}}, \quad\left\langle\left(\mu_{C}\right)_{i}^{\bar{j}}\right\rangle=\delta_{i}^{\bar{j}} \mu_{C} .
$$

The nonzero vacuum condensate $\left\langle\bar{C}_{2, \bar{j}} C_{2}^{i}\right\rangle=Z_{2}\left\langle\bar{Q}_{2, \bar{j}} Q_{2}^{i}\right\rangle=Z_{2} \mu_{C}^{2} \delta_{\bar{j}}^{i}=\left(\langle S\rangle / \mu_{C}\right) \delta_{\bar{j}}^{i}$ of these heavy constituent quarks is a pure quantum effect from the one-loop triangle diagram with the constituent quark fields $C_{2}$ and $\bar{C}_{2}$ contracted into their massive propagators with the masses $\mu_{C}$ and emitting two external gluino lines, this contribution realizes the Konishi anomaly.

Besides, by definition, all effects of evolution through the threshold region are already taken into account in (3.2), so that the quark terms in the Lagrangian are needed practically for calculations with the valence heavy quarks only. And finally, dots in (3.2) indicate other possible D-terms which are supposed to play no significant role in what follows.

To a large extent, the form of the Lagrangian in (3.2) is unique, once the main assumption about formation at the scale $\mu \sim \mu_{C}$ of massive constituent quarks with masses $\mu_{C}^{2}=\left\langle\bar{Q}_{2} Q_{2}\right\rangle=\left\langle\Pi_{2}\right\rangle$ and light pions with masses $m_{2}$ (and with all gluons remaining massless) is adopted. The only important non-trivial point, may be, is the non-zero value of the coefficient $\left(-N_{F}\right)$ in front of the second term in the superpotential $W_{Q}$. This was determined from the requirement that, until quark and/or gauge degrees of freedom are not integrated out, the vacuum value of the superpotential is not changed yet, in comparison with its original value at higher scales $\mu \gg \mu_{C}:\left\langle W_{Q}\right\rangle=\sum_{\text {flav }} m_{Q}(\mu)\left\langle(Q \bar{Q})_{\mu}\right\rangle=N_{F}\langle S\rangle$ (contributions of all three terms in $W_{Q}$ in (3.2) to $\left\langle W_{Q}\right\rangle$ are equal $N_{F}\langle S\rangle$ each, but the vacuum averages of the first and second terms in (3.2) cancel each other). 
The absolute value of $\left\langle\Pi_{2}\right\rangle=\left\langle\bar{Q}_{2} Q_{2}\right\rangle$ can be determined from the Konishi anomaly:

$$
\frac{1}{\left\langle\Pi_{2}\right\rangle}\left(\frac{\operatorname{det}\left\langle\Pi_{2}\right\rangle}{\Lambda_{Q}^{\mathrm{b}_{o}}}\right)^{1 / \bar{N}_{c}}\left\langle\bar{Q}_{2} Q_{2}\right\rangle=\langle S\rangle=\left(\frac{\operatorname{det}\left\langle\Pi_{2}\right\rangle}{\Lambda_{Q}^{\mathrm{b}_{o}}}\right)^{1 / \bar{N}_{c}} .
$$

Together with $m_{2}\left\langle\Pi_{2}\right\rangle=\langle S\rangle$, it follows from (3.4) (see (2.5-2.7)) that: $m_{2}=m_{Q} \equiv$ $m_{Q}\left(\mu=\Lambda_{Q}\right)$ and $\mu_{C}^{2}=\left\langle\Pi_{2}\right\rangle=\left\langle\bar{Q}_{2} Q_{2}\right\rangle=\langle\bar{Q} Q\rangle_{\mu=\Lambda_{Q}} \equiv \mathcal{M}_{\mathrm{ch}}^{2}$. 5

It is also useful to consider the evolution through the threshold region in more detail. At the scale $\mu=\mu_{1}$, there is no real distinction yet between the original light quarks $Q_{1}=Q\left(\mu=\mu_{1}\right)$ and $\bar{Q}_{1}=\bar{Q}\left(\mu=\mu_{1}\right)$ with the current masses $m_{1}=m_{Q}\left(\mu=\mu_{1}\right)$ and the (heavy at scales $\left.\mu<\mu_{2}\right)$ constituent quarks $C_{1}=C\left(\mu=\mu_{1}\right), \bar{C}_{1}=\bar{C}\left(\mu=\mu_{1}\right)$, because the large constituent quark mass $\mu_{C}$ "turns on and saturates" only after the evolution through the threshold region $\mu_{2}<\mu<\mu_{1}$. Similarly, there is no real distinction between the light composite field $(Q \bar{Q})\left(\mu=\mu_{1}\right)$ with its mass scale $\sim m_{1}$ and the pion field $\Pi_{1}=\Pi\left(\mu=\mu_{1}\right)$ (this is the pion $\Pi_{2}=\Pi\left(\mu=\mu_{2}\right)$ evolved back to $\left.\mu=\mu_{1}\right)$, with its mass $m_{2}$ at $\mu=\mu_{2}$ evolving back to the current quark mass $m_{1}$ at $\mu=\mu_{1}$. In essence, all these are the obvious matching conditions. They can be also used as an independent check that the form of $W_{Q}$ in (3.2) is self-consistent. After evolving back from $\mu=\mu_{2}$ to $\mu=\mu_{1}$, the difference between the composite field $\bar{Q} Q$ of heavy constituent quarks and the field $\Pi$ of the light pion disappears due to disappearance of the mass gap $\sim \mu_{C}$, so that two first terms in $W_{Q}$ cancel each other, while the last term evolves back into the original quark mass term.

But then, at $\mu<\mu_{1}$, the colorless light composite pions and colored heavy constituent quarks evolve differently through the threshold region $\mu_{2} \leq \mu \leq \mu_{1}$, and their Kahler terms acquire different renormalization factors. The renormalization factor $Z_{\pi}$ of pions is: from $\Pi_{1} \sim\left(Q_{1} \bar{Q}_{1}\right)$ with the mass $m_{1}$ at $\mu=\mu_{1}$ to $\Pi_{2}=Z_{\pi} \Pi_{1}$, with the mass $m_{2}$ at $\mu=\mu_{2}$, i.e.: $Z_{\pi}=m_{1} / m_{2}$. Similarly, the overall renormalization factor of quarks is: from $\left(C_{1}^{\dagger} C_{1}\right) \sim\left(Q_{1}^{\dagger} Q_{1}\right)$ with the mass $m_{1}$ at $\mu=\mu_{1}$ to $\left(C_{2}^{\dagger} C_{2}\right)=Z_{Q}\left(C_{1}^{\dagger} C_{1}\right)$, with the mass $\mu_{C}$ at $\mu=\mu_{2}$, i.e.: $Z_{Q}=m_{1} / \mu_{C}$.

Independently of (3.4), the absolute values of $m_{2}$ (the parameter $m_{2}$ will enter explicitly the lowest energy Lagrangian and will determine the observable pole masses of pions, $\left.M_{\pi} \sim m_{2}\right)$ and $\left\langle\Pi_{2}\right\rangle=\mu_{C}^{2}$ can be obtained from the following reasoning. Let us rewrite, say, the second term in the quark superpotential in (3.2) in terms of the quark fields $\left(Q_{1} \bar{Q}_{1}\right)$ normalized at $\mu=\mu_{1}$ and then, once more, in terms of $\left(Q_{\mu} \bar{Q}_{\mu}\right)$ normalized at running $\mu>\mu_{1}$ : 6

$$
\begin{gathered}
\left(\frac{\operatorname{det} \Pi_{2}}{\Lambda_{Q}^{\mathrm{b}_{o}}}\right)^{1 / \bar{N}_{c}}=Z_{\pi}^{N_{F} / \bar{N}_{c}}\left(\frac{\operatorname{det}\left(Q_{1} \bar{Q}_{1}\right)}{\Lambda_{Q}^{\mathrm{b}_{o}}}\right)^{1 / \bar{N}_{c}}= \\
\left(Z_{\pi} z_{Q}\left(\mu, \mu_{1}\right)\right)^{N_{F} / \bar{N}_{c}}\left(\frac{\operatorname{det}\left(Q_{\mu} \bar{Q}_{\mu}\right)}{\Lambda_{Q}^{\mathrm{b}_{\mathrm{o}}}}\right)^{1 / \bar{N}_{c}}=\left(Z_{\pi} z_{Q}\left(\Lambda_{Q}, \mu_{1}\right)\right)^{N_{F} / \bar{N}_{c}}\left(\frac{\operatorname{det}\left(Q_{\Lambda_{Q}} \bar{Q}_{\Lambda_{Q}}\right)}{\Lambda_{Q}^{\mathrm{b}_{\mathrm{o}}}}\right)^{1 / \bar{N}_{c}}
\end{gathered}
$$

${ }^{5}$ It is worth noting that the concrete form of the Kahler term $K_{\pi}$ of quantum pion fields $\pi_{\frac{i}{j}}$ in (3.2) should not be taken literally. Its only purpose is to show a typical scale of this Kahler term. For instance, one can replace it with the contribution $\sim \operatorname{Tr}\left(\mu_{C}^{\dagger} \mu_{C}\right)$ from the loop of constituent quarks, where the field $\left(\mu_{C}\right)_{i}^{\bar{j}}$ is given in (3.3). Finally, to determine the values of pion masses up to non-parametrical factors $\sim 1$, it is only important that both these forms of the pion Kahler term have the same scale $\left\langle K_{\pi}\right\rangle \sim \mathcal{M}_{\mathrm{ch}}^{2}$. For similar reasons, we neglect possible additional dependence of $Z_{2}$ - factors entering the Kahler term of the constituent quark in (3.2) on the quantum pion fields $\pi / \mathcal{M}_{\mathrm{ch}}$.

${ }^{6}$ It is worth noting that this is only a change of notations, not a real evolution to another scale. 
Clearly, at running $\mu_{1} \leq \mu \leq \Lambda_{Q}$, the coefficient in front of the field $\left(Q_{\mu} \bar{Q}_{\mu}\right)$ depends explicitly on the running scale $\mu$ through the quark perturbative renormalization factor $z_{Q}\left(\mu, \mu_{1}\right)$, while $Z_{\pi}$ is independent of $\mu$. So, to find the value of $Z_{\pi}$, we have to fix the normalization at some definite value of $\mu$. The only distinguished point is $\mu=$ $\Lambda_{Q}$ in a sense that this term in the superpotential, being expressed though the fields $\left(Q_{\mu=\Lambda_{Q}} \bar{Q}_{\mu=\Lambda_{Q}}\right)$ normalized at $\Lambda_{Q}$, should have the coefficient which depends on $\Lambda_{Q}$ only. From this, it follows:

$$
\begin{gathered}
Z_{\pi}=\frac{m_{1}=m_{Q}\left(\mu=\mu_{1}\right)}{m_{2}}=z_{Q}^{-1}\left(\Lambda_{Q}, \mu_{1}\right) \equiv z_{Q}^{-1} \gg 1, \quad \mu_{1} \sim \mu_{C} \ll \Lambda_{Q}, \\
\mu_{C}^{2}=\left\langle\Pi_{2}\right\rangle=\left\langle\bar{Q}_{2} Q_{2}\right\rangle=\langle\bar{Q} Q\rangle_{\mu=\Lambda_{Q}} \equiv \mathcal{M}_{\mathrm{ch}}^{2}, \quad m_{2}=m_{Q}\left(\mu=\Lambda_{Q}\right) \equiv m_{Q}, \\
Z_{2}=\frac{\Lambda_{o}}{\mu_{C}}=\left(\frac{\mathcal{M}_{\mathrm{ch}}}{\Lambda_{Q}}\right)^{\mathrm{b}_{\mathrm{o}} / \overline{\mathrm{N}}_{\mathrm{c}}}=\frac{m_{Q}}{\mathcal{M}_{\mathrm{ch}}}, \quad Z_{Q}=\frac{m_{1}}{\mu_{C}}=\frac{m_{2}}{\mu_{C}} \frac{m_{1}}{m_{2}}=\frac{m_{Q}}{\mathcal{M}_{\mathrm{ch}}} \frac{m_{1}}{m_{2}}=Z_{2} Z_{\pi}=Z_{2} z_{Q}^{-1},
\end{gathered}
$$

where $z_{Q}\left(\Lambda_{Q}, \mu=\mu_{1}\right) \ll 1$ is the standard perturbative renormalization factor of the massless quark describing its evolution from $\mu=\Lambda_{Q}$ down to $\mu=\mu_{1}$ (in the conformal window it is known explicitly: $\left.z_{Q}=z_{Q}\left(\Lambda_{Q}, \mu_{1}\right)=\left(\mu_{1} / \Lambda_{Q}\right)^{\mathrm{b}_{\circ} / \mathrm{N}_{\mathrm{F}}} \ll 1\right)$.

On the whole, the evolution of the current quark mass in the interval $\mu_{2} \leq \mu \leq \Lambda_{Q}$ looks as follows. At $\mu=\Lambda_{Q}$ the current quark mass is $m_{Q} \equiv m_{Q}\left(\mu=\Lambda_{Q}\right)$. At smaller $\mu$ it runs with the perturbative $z_{Q}^{(\mu)}=z_{Q}\left(\Lambda_{Q}, \mu\right)$-factor, $m_{Q}(\mu)=m_{\Lambda_{Q}} / z_{Q}^{(\mu)} \gg m_{Q}$, so that $m_{1} \equiv m_{Q}\left(\mu=\mu_{1}\right)=z_{Q}^{-1} m_{Q}$. In the threshold region $\mu_{2}<\mu<\mu_{1}$ it runs so that ( at $\mu<\mu_{1}$ the current quark mass can be understood more properly as the pion mass): $m_{1} \equiv m_{Q}\left(\mu=\mu_{1}\right) \rightarrow m_{2} \equiv m_{Q}\left(\mu=\mu_{2}\right), m_{2}=Z_{\pi}^{-1} m_{1}$. And at $\mu \ll \mu_{2}$ the current quark mass $m_{2}$ does not run any more. Using that $Z_{\pi}=z_{Q}^{-1}$ from (3.6), it is seen that, evolving through the threshold region from $\mu=\mu_{1}$ down to $\mu=\mu_{2}$, the current quark mass returns back to its value at $\mu=\Lambda_{Q}: m_{2}=Z_{\pi}^{-1} m_{1}=Z_{\pi}^{-1}\left(z_{Q}^{-1} m_{Q}\right)=m_{Q}$. As for the constituent quark mass $\mu_{C}$, it originates in the threshold region $\mu \sim \mu_{C}$ due to an existence of the coherent quark condensate, $\mu_{C}^{2}=\left\langle\bar{Q}_{2} Q_{2}\right\rangle=\mathcal{M}_{\mathrm{ch}}^{2}$, and it stops the further RG-evolution of the constituent quark and pion fields at $\mu<\mu_{C}=\mathcal{M}_{\text {ch. }}$. The self-consistency of this scenario requires that $\mu_{C}=\mathcal{M}_{\mathrm{ch}}$ be larger than $m_{Q}^{\text {pole }}$, because otherwise the massless conformal regime will stop before at $\mu=m_{Q}^{\text {pole }}$, i.e. quarks will be in the HQ (heavy quark) phase and the coherent quark condensate can't be formed in this case. In the case considered, with $3 N_{c} / 2<N_{F}<3 N_{c}$,

$$
\frac{m_{Q}^{\text {pole }}}{\Lambda_{Q}} \equiv \frac{m_{Q}\left(\mu=m_{Q}^{\text {pole }}\right)}{\Lambda_{Q}}=\frac{m_{Q}}{\Lambda_{Q}}\left(\frac{\Lambda_{Q}}{m_{Q}^{\text {pole }}}\right)^{\mathrm{b}_{\circ} / N_{\mathrm{F}}}=\left(\frac{m_{Q}}{\Lambda_{Q}}\right)^{N_{F} / 3 N_{c}}=\frac{\Lambda_{Y M}}{\Lambda_{Q}} \ll \frac{\mu_{C}}{\Lambda_{Q}}=\frac{\mathcal{M}_{\mathrm{ch}}}{\Lambda_{Q}}
$$

so that this is self-consistent.

Let us dwell now on the evolution of the Wilsonian coupling $\alpha_{W}(\mu)$ in the interval $\mu_{2}<\mu<\Lambda_{Q}$. Let us recall first its standard perturbative evolution in the interval $\mu_{1}<\mu<\Lambda_{Q}$ :

$$
\delta\left(\frac{2 \pi}{\alpha_{W}(\mu)}\right)=\left\{3 N_{c} \ln \frac{\mu}{\Lambda_{Q}}-N_{F} \ln \frac{\mu}{\Lambda_{Q}}\right\}+\left\{N_{F} \ln \frac{1}{z_{Q}(\mu)}\right\}
$$


where the first two terms are the one-loop contributions of massless gluons and quarks, while the last term describes higher-loop effects from massless quarks [5]. In the conformal window $3 N_{c} / 2<N_{F}<3 N_{c}$ the explicit form of the quark renormalization factor $z_{Q}(\mu)$ is known at $\mu<\Lambda_{Q}: z_{Q}(\mu) \equiv z_{Q}\left(\Lambda_{Q}, \mu\right)=\left(\mu / \Lambda_{Q}\right)^{\mathrm{b}_{\mathrm{o}} / \mathrm{N}_{\mathrm{F}}} \ll 1$. Then, the above three parametrically large logarithmic terms in (3.7) cancel each other. This describes the standard effect that the perturbative coupling freezes in the conformal regime at $\alpha^{*}=$ $O(1)$, i.e. it remains nearly the same as it was at $\mu=\Lambda_{Q}$, as $\alpha\left(\mu=\Lambda_{Q}\right)$ is already close to $\alpha^{*}$, by definition of $\Lambda_{Q}$.

This perturbative form (3.7) can be used down to $\mu>\mu_{1}$. Now, on account of additional contributions from the threshold region $\mu_{2}<\mu<\mu_{1}$, the coupling $\alpha\left(\mu, \Lambda_{L}\right)$ at $\mu<\mu_{2}$ looks as (the number $2 \pi / N_{c} \alpha\left(\mu=\Lambda_{Q}\right)$ is considered as $O(1)$ and is neglected in comparison with the large logarithm):

$$
\begin{gathered}
\frac{2 \pi}{\alpha_{W}\left(\mu<\mu_{2}, \Lambda_{L}\right)}=\left\{\frac{2 \pi}{\alpha\left(\mu<\mu_{2}, \Lambda_{L}\right)}-N_{c} \ln \left(\frac{1}{\mathrm{~g}^{2}\left(\mu,\left\langle\Lambda_{L}\right\rangle\right)}\right)\right\}= \\
=\left\{3 N_{c} \ln \frac{\mu}{\Lambda_{Q}}-\ln \left(\frac{\operatorname{det}\left(\mu_{C}\right)_{i}^{\bar{j}}}{\Lambda_{Q}^{N_{F}}}\right)+N_{F}\left(\ln \frac{1}{z_{Q}}+\ln \frac{1}{Z_{Q}}\right)\right\} .
\end{gathered}
$$

Here:

a) the first term in the curly brackets in (3.8) is due to contributions of massless gluons; b) in the second term in the curly brackets the one-loop term from colored quarks stops now its evolution at their constituent mass $\left(\mu_{C}\right) \frac{i}{j}$, see (3.3), i.e. with surviving light pion fields $\pi_{j}^{i}$ still living at lower energies; besides, in addition to the previous term $\ln \left(1 / z_{Q}\right), z_{Q} \equiv z_{Q}\left(\Lambda_{Q}, \mu_{1}\right)$, which describes the standard smooth perturbative evolution from $\mu=\Lambda_{Q}$ down to $\mu_{1}$, there appeared the last term $\ln \left(1 / Z_{Q}\right)$ which is due to the additional (non-standard) evolution of the colored constituent quark in the threshold region $\mu_{2} \leq \mu \leq \mu_{1}$.

Numerically (i.e. neglecting the quantum pion fields $\pi \frac{i}{j} / \mathcal{M}_{\mathrm{ch}}$ and replacing $\operatorname{det} \Pi_{2}$ by its vacuum value $\mathcal{M}_{\mathrm{ch}}^{2 N_{F}}$ ), the first three terms in the r.h.s. of (3.8) still cancel each other. So, the parametrically large value of $1 / \alpha_{W}\left(\mu<\mu_{2}\right)$ (i.e. the weak coupling) originates from the parametrically large $\ln \left(1 / Z_{Q}\right)$ threshold contribution only. In other words, the strong evolution of the coupling $\alpha(\mu)$ in the threshold region $\mu_{2}<\mu<\mu_{1}$ decreases it from the $O(1)$ value at $\mu=\mu_{1}$ to a logarithmically small value $\alpha\left(\mu_{2}\right) \sim \alpha_{W}\left(\mu_{2}\right) \sim$ $1 / \ln \left(\Lambda_{Q} / \mathcal{M}_{\mathrm{ch}}\right)$ at $\mu=\mu_{2}$.

Substituting into (3.8) the value of $Z_{Q}$ from (3.6) and $\operatorname{det}\left(\mu_{C}\right)_{i}^{\bar{j}}$ from (3.3), one can write finally the Yang-Mills coupling as :

$$
\begin{gathered}
\frac{2 \pi}{\alpha_{W}\left(\mu<\mu_{2}\right)}=\left\{\frac{2 \pi}{\alpha\left(\mu, \Lambda_{L}\right)}-N_{c} \ln \frac{1}{g^{2}\left(\mu,\left\langle\Lambda_{L}\right\rangle\right)}\right\}=3 N_{c} \ln \frac{\mu}{\Lambda_{L}}, \\
\Lambda_{L}=\left(\frac{\operatorname{det} \Pi_{2}}{\Lambda_{Q}^{\mathrm{b}_{o}}}\right)^{1 / 3 \bar{N}_{c}}, \quad \Lambda_{Y M} \equiv\left\langle\Lambda_{L}\right\rangle=\left(\frac{\mathcal{M}_{\mathrm{ch}}^{2 N_{F}}}{\Lambda_{Q}^{\mathrm{b}_{\mathrm{o}}}}\right)^{1 / 3 \bar{N}_{c}} .
\end{gathered}
$$

Let us emphasize (this will be important for us in section 7) that the explicit value of the quark perturbative renormalization factor $z_{Q}=z_{Q}\left(\Lambda_{Q}, \mu_{1} \sim \mathcal{M}_{\mathrm{ch}}\right)$ is not really 
needed to obtain (3.9), because $z_{Q}$ cancels exactly in (3.8), independently of its explicit form (and $Z_{2}$ also).

Now, at lower scales $\mu<\mu_{2}$, if we are not interested in calculations with the valence quarks, the fields of heavy constituent quarks can be integrated out. 7

This will result in simply omitting in (3.2) all terms containing the quark fields (let us recall that the quark loop contributions to the gauge coupling have been taken into account already in (3.8)). Besides, the pion fields $\Pi_{2}$ (and masses $m_{2}$ ) do not evolve any more at $\mu<\mu_{2}$, so that $\mathcal{M}_{\mathrm{ch}}$ from $\mathcal{M}_{\mathrm{ch}}{ }^{2}=\left\langle\Pi_{2}\right\rangle$ and $m_{2}$ become the low energy constant observables at $\mu \ll \mathcal{M}_{\text {ch }}$ (the pion pole mass will be $\sim m_{2}$ and $\mathcal{M}_{\text {ch }}=\langle S\rangle / m_{2}$, or $\langle S\rangle$ itself, are connected with tensions of BPS domain walls between different vacua [9]). Therefore, the only remaining evolution in the interval $\Lambda_{Y M} \ll \mu \ll \mathcal{M}_{\text {ch }}$ is the standard (weak coupling) perturbative logarithmic evolution of massless gluons, so that in this range of scales the Lagrangian takes the form (from now on, to simplify the notations, we substitute: $\Pi_{2} \equiv \Pi$, and $m_{2}=m_{Q} \equiv m_{Q}\left(\mu=\Lambda_{Q}\right)$, see also the footnote 3 about the R-charge) :

$$
\begin{gathered}
L=\int d^{2} \theta d^{2} \bar{\theta}\left\{\operatorname{Tr} \sqrt{\Pi^{\dagger} \Pi}\right\}+\int \mathrm{d}^{2} \theta\left\{-\frac{2 \pi}{\alpha\left(\mu, \Lambda_{\mathrm{L}}\right)} \mathrm{S}-\mathrm{N}_{\mathrm{F}}\left(\frac{\operatorname{det} \Pi}{\Lambda_{\mathrm{Q}}^{\mathrm{b}_{\mathrm{o}}}}\right)^{1 / \overline{\mathrm{N}}_{\mathrm{c}}}+\mathrm{m}_{\mathrm{Q}} \operatorname{Tr} \Pi\right\}, \\
\Lambda_{L}=\left(\frac{\operatorname{det} \Pi}{\Lambda_{Q}^{\mathrm{b}_{\mathrm{o}}}}\right)^{1 / 3 \bar{N}_{c}}, \quad \Lambda_{Y M} \ll \mu \ll \mathcal{M}_{\mathrm{ch}}
\end{gathered}
$$

Lowering the scale $\mu$ down to $\mu<\Lambda_{Y M}$ and integrating out all gauge degrees of freedom, except for the one whole field $S$ itself (this leaves behind a large number of gluonia with masses $M_{\mathrm{gl}} \sim \Lambda_{Y M}$ ), one obtains the $\mathrm{VY}$-form :

$$
\begin{gathered}
L=\int d^{2} \theta d^{2} \bar{\theta}\left\{\operatorname{Tr} \sqrt{\Pi^{\dagger} \Pi}\right\}+(\mathrm{D} \text { terms of the field } \mathrm{S})+ \\
+\int d^{2} \theta\left\{-N_{c} S\left(\ln \frac{S}{\Lambda_{L}^{3}}-1\right)-N_{F}\left(\frac{\operatorname{det} \Pi}{\Lambda_{Q}^{\mathrm{b}_{\mathrm{o}}}}\right)^{1 / \bar{N}_{c}}+m_{Q} \operatorname{Tr} \Pi\right\}, \mu<\Lambda_{\mathrm{YM}} .
\end{gathered}
$$

Finally, at lower energies $\mu \ll \Lambda_{Y M}$, after integrating out the last gluonium field $S$ (with its mass scale $\sim \Lambda_{Y M}$ ), one obtains the Lagrangian of pions:

$$
L=\int d^{2} \theta d^{2} \bar{\theta}\left\{\operatorname{Tr} \sqrt{\Pi^{\dagger} \Pi}\right\}+
$$

7 Because quarks are confined, this leaves behind a large number of heavy quarkonia, both mesons and baryons, with masses $M_{\text {meson }} \sim \mathcal{M}_{\text {ch }}$ and $M_{\text {baryon }} \sim N_{c} \mathcal{M}_{\mathrm{ch}}$, built from non-relativistic (and weakly confined, the string tension is $\sqrt{\sigma} \sim \Lambda_{Y M} \ll \mathcal{M}_{\mathrm{ch}}$ ) constituent quarks with masses $\mu_{C}=\mathcal{M}_{\mathrm{ch}}$. Indeed, the characteristic distance between the non-relativistic quarks in the bound state is the Bohr radius: $R_{B} \sim 1 / p_{B}$, where $p_{B}$ is the Bohr momentum $p_{B} \sim \alpha\left(\bar{\mu} \simeq p_{B}\right) \mathcal{M}_{\mathrm{ch}}$. Supposing that $p_{B} \ll \mathcal{M}_{\mathrm{ch}}$, this requires $\alpha\left(\bar{\mu} \ll \mathcal{M}_{\text {ch }}\right) \ll 1$. But indeed (see above), in this region $\Lambda_{Y M} \ll \mu \ll \mathcal{M}_{\text {ch }}$ the coupling is already logarithmically small, $\alpha(\mu) \sim 1 / \ln \left(\mu / \Lambda_{Y M}\right) \ll 1$. So, the nonrelativistic regime is self-consistent $\left(\alpha(\mu)\right.$ becomes $O(1)$ only at much smaller distances $R_{\mathrm{ch}} \sim 1 / \mathcal{M}_{\mathrm{ch}} \ll R_{B}$, while confinement effects begin to be important only at much larger distances $R_{\text {conf }} \sim 1 / \Lambda_{Y M} \gg R_{B}$ ). 


$$
+\int d^{2} \theta\left\{-\bar{N}_{c}\left(\frac{\operatorname{det} \Pi}{\Lambda_{Q}^{\mathrm{b}_{\mathrm{o}}}}\right)^{1 / \bar{N}_{c}}+m_{Q} \operatorname{Tr} \Pi\right\}, \mu \ll \Lambda_{\mathrm{YM}} .
$$

This describes weakly interacting pions with the smallest masses $m_{\pi} \sim m_{Q} \cdot 8$

So, this is the end of this story. 9

\section{Dual theory . Definition}

The Lagrangian of the dual theory (at the scale $\mu \sim \Lambda_{Q}$ ) is taken in the form [1] :

$$
\begin{gathered}
\bar{L}=\int d^{2} \theta d^{2} \bar{\theta}\left\{\operatorname{Tr}\left(q^{\dagger} e^{\bar{V}} q+\bar{q}^{\dagger} e^{-\bar{V}} \bar{q}\right)+\frac{1}{\left(\mu_{q}^{\prime}\right)^{2}} \operatorname{Tr}\left(\mathrm{M}^{\dagger} \mathrm{M}\right)\right\}+ \\
\int d^{2} \theta\left\{-\frac{2 \pi}{\bar{\alpha}\left(\mu, \Lambda_{q}\right)} \bar{s}+\frac{1}{\mu_{q}} \operatorname{Tr}(\overline{\mathrm{q}} \mathrm{M} \mathrm{q})+\overline{\mathrm{m}}_{\mathrm{Q}}(\mu) \operatorname{Tr} \mathrm{M}\right\}+\text { h.c. }, \quad \overline{\mathrm{s}}=\overline{\mathrm{w}}_{\alpha}^{2} / 32 \pi^{2} .
\end{gathered}
$$

Here: $\bar{a}(\mu)=\bar{N}_{c} \bar{\alpha}(\mu) / 2 \pi$ is the running dual coupling (with its scale parameter $\Lambda_{q}$ ), $a_{f}(\mu)=N_{F} f^{2}(\mu) / 4 \pi$ will be its running Yukawa coupling (with its scale parameter $\Lambda_{f}$ ) with $f\left(\mu=\Lambda_{Q}\right) \sim \mu_{q}^{\prime} / \mu_{q}, \bar{w}_{\alpha}$ is the dual gluon field strength. This theory has the exact $S U\left(\bar{N}_{c}=N_{F}-N_{c}\right)$ gauge symmetry, while in the chiral limit $\bar{m}_{Q} \rightarrow 0$ the global symmetries are the same as in the direct theory. Under these symmetries the dual quarks and mesons $\mathrm{M}$ (mions) transform as:

$$
\begin{gathered}
q: \quad\left(\bar{N}_{F}\right)_{L}^{\mathrm{fl}} \times(0)_{R}^{\mathrm{fl}} \times\left(N_{c} / \bar{N}_{c}\right)_{B} \times\left(N_{c} / N_{F}\right)_{R}, \\
\bar{q}: \quad(0)_{L}^{\mathrm{fl}} \times\left(N_{F}\right)_{R}^{\mathrm{fl}} \times\left(-N_{c} / \bar{N}_{c}\right)_{B} \times\left(N_{c} / N_{F}\right)_{R}, \\
\mathrm{M}: \quad\left(N_{F}\right)_{L}^{\mathrm{fl}} \times\left(\bar{N}_{F}\right)_{R}^{\mathrm{fl}} \times(1)_{B} \times\left(2 \bar{N}_{c} / N_{F}\right)_{R} .
\end{gathered}
$$

The mion fields $\mathrm{M}_{\frac{i}{j}}^{i}$ in (4.1) are defined as pointlike ones. This is unlike the pion fields $\Pi \frac{i}{j}$ of the direct theory, which appear as light pointlike fields only at energies below the scale of chiral flavor symmetry breaking, $\mu<\mu_{C}=\mathcal{M}_{\text {ch }}$. At higher scales $\mu \gg \mathcal{M}_{\text {ch }}$ they, strictly speaking, can't be used at all (or, at best, can be resolved as composite fields of two current quarks).

To match parameters of the direct and dual theories (see below), the normalizations at $\mu=\Lambda_{Q}$ are taken as:

$$
\left\langle\mathrm{M}_{\bar{j}}^{i}\right\rangle_{\mu=\Lambda_{Q}}=\mathcal{M}_{\mathrm{ch}}^{2} \delta \frac{i}{j}, \quad \bar{m}_{Q}\left(\mu=\Lambda_{Q}\right)=m_{Q}\left(\mu=\Lambda_{Q}\right) \equiv m_{Q}
$$

Besides, to match the values of gluino condensates, the scale parameter $\Lambda_{q}$ has to be taken as [3] :

$$
\Lambda_{\mathrm{q}}^{\overline{\mathrm{b}}_{\mathrm{o}}}=(-1)^{\bar{N}_{c}}\left(\mu_{q}^{N_{F}} / \Lambda_{Q}^{\mathrm{b}_{\mathrm{o}}}\right) \quad \rightarrow \quad\langle S\rangle=\langle-\bar{s}\rangle, \quad \overline{\mathrm{b}}_{\mathrm{o}}=\left(3 \overline{\mathrm{N}}_{\mathrm{c}}-\mathrm{N}_{\mathrm{F}}\right)
$$

\footnotetext{
8 The vacuum value $\left\langle\Pi_{\frac{i}{j}}\right\rangle=\mathcal{M}_{\mathrm{ch}}^{2} \delta_{\frac{i}{j}}$ recalls the scale $\mu_{C}=\mathcal{M}_{\mathrm{ch}}$ at which they were formed and so determines their "internal hardness", i.e. the scale up to which they behave as pointlike particles.

9 A short discussion of external anomalies (the 't Hooft triangles) is transferred to the appendix.
} 


\section{Dual theory with $\mu_{q}=\Lambda_{Q}$. Conformal window}

With this choice, $\left|\Lambda_{q}\right|=\Lambda_{Q}$, see (4.4). In essence, this is the only natural value for $\mu_{q}$, from a viewpoint of the direct theory. At $\mu_{q} \ll \Lambda_{Q}$ the value of $\left|\Lambda_{q}\right|$ will be either artificially small (at $N_{F}>3 N_{c} / 2$ ), or artificially large (at $N_{F}<3 N_{c} / 2$ ), see (4.4). At $\Lambda_{f} \sim\left|\Lambda_{q}\right|=\Lambda_{Q},\left(\mu_{q}^{\prime} \sim \mu_{q}\right)$, the dual theory (which, self-consistently by itself, is considered to be in the UV-free logarithmic regime at $\mu \gg \Lambda_{Q}$, with $a_{f}(\mu)<\bar{a}(\mu)$ at $\mu \gg \Lambda_{Q}$ ) enters, simultaneously with the direct one, the superconformal regime at $\mu \sim \Lambda_{Q}$, with frozen couplings : $\bar{a}(\mu) \rightarrow \bar{a}^{*}$ and $a_{f}(\mu) \rightarrow a_{f}^{*}$. The dynamical dimensions of chiral superfields are determined here by their R-charges, $\mathrm{D}=3|\mathrm{R}| / 2$, so that, for instance, the distance dependence of the two-point correlators $\left\langle\left\{\bar{Q}_{\bar{j}} Q^{i}(x)\right\}^{\dagger}, \bar{Q}_{\bar{l}} Q^{k}(0)\right\rangle$ and $\left\langle\left\{M_{\bar{j}}^{i}\right\}^{\dagger}(x), M_{\bar{l}}^{k}(0)\right\rangle$ is the same, etc. [1]. Besides, all 't Hooft triangles are matched [1. At present, no indication of possible differences between the direct and dual theories is known in this perturbative superconformal regime. So, let us go to lower energies where the physical scales originating from the chiral symmetry breaking begin to reveal itself. What happens in the direct theory when reaching its highest physical scale $\mu_{H} \sim \mu_{C}=\mathcal{M}_{\text {ch }}$ was described above in section 3 .

In the dual theory and in the case considered, the highest physical scale $\mu_{H}$ is determined by the constituent mass $\bar{\mu}_{C}$ of dual quarks, i.e. by the value of their coherent condensate: $\mu_{H}=\bar{\mu}_{C}=|\langle\bar{q} q\rangle|_{\mu=\Lambda_{Q}}^{1 / 2}=\left(m_{Q} \Lambda_{Q}\right)^{1 / 2}$, as this is parametrically larger in the conformal window $3 N_{c} / 2<N_{F}<3 N_{c}$ than the pole mass $m_{q}^{\text {pole }}$ of dual quarks $\left(m_{q}(\mu)\right.$ is the running current mass of dual quarks, $m_{q}=m_{q}\left(\mu=\Lambda_{Q}\right)=\mathcal{M}_{\mathrm{ch}}^{2} / \Lambda_{Q}, \gamma_{q}=\overline{\mathrm{b}}_{\mathrm{o}} / \mathrm{N}_{\mathrm{F}}=$

$$
\begin{aligned}
& \left.\left(3 \overline{\mathrm{N}}_{\mathrm{c}}-\mathrm{N}_{\mathrm{F}}\right) / \mathrm{N}_{\mathrm{F}}, \Lambda_{\mathrm{YM}}=\left(\Lambda_{Q}^{\mathrm{b}_{\mathrm{o}}} \operatorname{det} \mathrm{m}_{\mathrm{Q}}\right)^{1 / 3 \mathrm{~N}_{\mathrm{c}}}\right): \\
& \frac{\bar{\mu}_{C}}{\Lambda_{Q}}=\left(\frac{m_{Q}}{\Lambda_{Q}}\right)^{1 / 2} \gg \frac{m_{q}^{\text {pole }}}{\Lambda_{Q}}, \quad \frac{m_{q}^{\text {pole }}}{\Lambda_{Q}}=\frac{m_{q}\left(\mu=m_{q}^{\text {pole }}\right)}{\Lambda_{Q}}=\frac{\mathcal{M}_{\mathrm{ch}}^{2}}{\Lambda_{Q}^{2}}\left(\frac{\Lambda_{Q}}{m_{q}^{\text {pole }}}\right)^{\gamma_{q}}=\frac{\Lambda_{Y M}}{\Lambda_{Q}} .
\end{aligned}
$$

This shows that, similarly to the direct theory, the dual theory is also in the same (dual) DC - phase here, with appearance $N_{F}^{2}$ dual pions $\mathrm{N}_{\mathrm{i}}^{\overline{\mathrm{j}}}$ (nions) and the large constituent masses $\bar{\mu}_{C}=\left(m_{Q} \Lambda_{Q}\right)^{1 / 2}$ of dual quarks when $\mu$ crosses the corresponding threshold region: $\bar{\mu}_{2}=\bar{\mu}_{C} /($ several $) \leq \mu \leq \bar{\mu}_{1}=$ (several) $\bar{\mu}_{\mathrm{C}}$. And similarly, all dual gluons also remain massless at the same time. Therefore, the pattern of evolution through the threshold region is universal, if either direct or dual theories are in the same DC - phase. So, using the same reasonings as those described above in section 3 and making some simple substitutions of direct parameters by dual ones, one obtains the effective dual Lagrangian at $\mu=\bar{\mu}_{2}$ in the form (the meson and quark fields are normalized at $\mu=\Lambda_{Q}$ in $(5.1))$ :

$$
\begin{aligned}
& \bar{L}=\int d^{2} \theta d^{2} \bar{\theta}\left\{\frac{z_{M}}{\Lambda_{Q}^{2}} \operatorname{Tr}\left(\mathrm{M}^{\dagger} \mathrm{M}\right)+\operatorname{Tr} \sqrt{\mathrm{N}^{\dagger} \mathrm{N}}+\overline{\mathrm{Z}}_{2} \operatorname{Tr}\left(\mathrm{q}^{\dagger} \mathrm{e}^{\overline{\mathrm{V}}} \mathrm{q}+\overline{\mathrm{q}}^{\dagger} \mathrm{e}^{-\overline{\mathrm{V}}_{\overline{\mathrm{q}}}}\right)\right\}+ \\
& +\int d^{2} \theta\left\{-\frac{2 \pi}{\bar{\alpha}\left(\bar{\mu}_{2}\right)} \bar{s}+W_{q}\right\}, \quad W_{q}=\frac{1}{\Lambda_{Q}} \operatorname{Tr}(\mathrm{MN})+m_{Q} \operatorname{Tr} \mathrm{M}+ \\
& +\left(\frac{\operatorname{det} \mathrm{N}}{\Lambda_{\mathrm{q}}^{\overline{\mathrm{b}}_{\mathrm{o}}}}\right)^{1 / N_{c}}\left[\operatorname{Tr}\left(\overline{\mathrm{q}}^{-1} \mathrm{q}\right)-\mathrm{N}_{\mathrm{F}}\right], \quad \overline{\mathrm{Z}}_{2}=\left(\frac{\bar{\mu}_{\mathrm{C}}}{\Lambda_{\mathrm{Q}}}\right)^{\overline{\mathrm{b}}_{\mathrm{o}} / \mathrm{N}_{\mathrm{c}}}=\left(\frac{\mathrm{m}_{\mathrm{q}}}{\bar{\mu}_{\mathrm{C}}}\right)
\end{aligned}
$$




$$
\left\langle\mathrm{M}_{\bar{j}}^{i}\right\rangle=\mathcal{M}_{\mathrm{ch}}^{2} \delta \frac{i}{j}, \quad\left\langle\mathrm{~N}_{i}^{\bar{j}}\right\rangle=\left\langle\bar{q}^{\bar{j}} q_{i}\right\rangle=-\bar{\mu}_{C}^{2} \delta_{i}^{\bar{j}}=-m_{Q} \Lambda_{Q} \delta_{i}^{\bar{j}}, \quad m_{q}=\mathcal{M}_{\mathrm{ch}}^{2} / \Lambda_{Q} .
$$

The factor $z_{M} \equiv z_{M}\left(\Lambda_{Q}, \bar{\mu}_{1}\right) \gg 1$ in (5.1) is the standard perturbative renormalization factor of mion fields $\mathrm{M}$ in the interval $\bar{\mu}_{1}<\mu<\Lambda_{Q}$ (the fields $\mathrm{M}, \mathrm{N}$ and the dual quarks are frozen and do not evolve any more at $\mu<\bar{\mu}_{2}$; besides, like the gluon fields, the mion fields $\mathrm{M}$ have no non-standard evolution in the threshold region; and finally, here and everywhere below we neglect, as in sections 2 and 3, the dependence of the renormalization factors $z_{M}$ and $\bar{Z}_{2}$ on the quantum mion and nion fields $m / \mathcal{M}_{\mathrm{ch}}$ and $n / \bar{\mu}_{C}$, as this will influence the particle mass values by non-parametric factors $\sim 1$ only, see also the footnote 5) :

$$
z_{M} \equiv z_{M}\left(\Lambda_{Q}, \bar{\mu}_{1}\right)=\left(\frac{\bar{\mu}_{1}}{\Lambda_{Q}}\right)^{\gamma_{M}}=1 / z_{q}^{2}, \quad z_{q} \equiv z_{q}\left(\Lambda_{Q}, \bar{\mu}_{1}\right)=\left(\frac{\bar{\mu}_{1}}{\Lambda_{Q}}\right)^{\overline{\mathrm{b}}_{\mathrm{o}} / \mathrm{N}_{\mathrm{F}}} \ll 1,
$$

where $z_{q}$ is the renormalization factor of the massless dual quarks due to the standard perturbative evolution from $\mu=\Lambda_{Q}$ down to $\bar{\mu}_{1}=$ (several) $\bar{\mu}_{\mathrm{C}}$.

And analogously to the direct theory, the factor $\bar{Z}_{2}$ in (5.1) is the overall renormalization factor of the dual quark due to its evolution from $\mu=\Lambda_{Q}$ down to $\mu=\bar{\mu}_{2}=$ $\bar{\mu}_{C} /$ (several). It can be written in the form: $\bar{Z}_{2}=z_{q} \bar{Z}_{q}$, where $z_{q}$ is due to the standard perturbative evolution in the interval $\bar{\mu}_{1}<\mu<\Lambda_{Q}$, while $\bar{Z}_{q}$ is due to the additional nonstandard evolution in the threshold region $\bar{\mu}_{2}=\bar{\mu}_{C} /$ (several) $<\mu<\bar{\mu}_{1}=($ several $) \bar{\mu}_{\mathrm{C}}$.

The heavy constituent dual quarks decouple at $\mu<\bar{\mu}_{2}$, and there remain the mions $\mathrm{M}$ and nions $\mathrm{N}$ and the pure gauge $S U\left(\bar{N}_{c}\right)$ dual theory. As for its inverse coupling $1 / \bar{\alpha}(\mu)$, one obtains, similarly to the direct theory, that it increases from its frozen value $1 / \bar{\alpha}^{*}=O(1)$ at $\mu=\bar{\mu}_{1}$ to a logarithmically large value at $\mu=\bar{\mu}_{2}$, due to the additional large renormalization factor $\bar{Z}_{q}$ of constituent dual quarks. The whole evolution from $\mu=\left|\Lambda_{q}\right|$ down to $\mu<\bar{\mu}_{2}$ results in:

$$
\begin{gathered}
\frac{2 \pi}{\bar{\alpha}\left(\mu<\bar{\mu}_{2}, \bar{\Lambda}_{L}\right)}=\left\{3 \bar{N}_{c} \ln \frac{\mu}{\Lambda_{q}}+\bar{N}_{c} \ln \frac{1}{\bar{g}^{2}\left(\mu,\left\langle\bar{\Lambda}_{L}\right\rangle\right)}\right\}-\left\{\ln \left(\frac{\operatorname{det}\left(\bar{\mu}_{C}\right)_{\bar{j}}^{i}}{\Lambda_{q}^{N_{F}}}\right)-N_{F} \ln \frac{1}{\bar{Z}_{2}}\right\} \\
\left(\bar{\mu}_{C}\right)_{\bar{j}}^{i}=\frac{1}{\bar{Z}_{2}}\left(\frac{\operatorname{det} \mathrm{N}}{\Lambda_{\mathrm{q}}^{\overline{\mathrm{b}}_{\mathrm{o}}}}\right)^{1 / N_{c}}\left(\mathrm{~N}^{-1}\right)_{\bar{j}}^{i}
\end{gathered}
$$

where $\left(\bar{\mu}_{C}\right)_{\bar{j}}^{i}$ is the constituent mass of dual quarks, see $(5.1)$.

Therefore, one obtains from (5.3) that the scale parameter $\bar{\Lambda}_{L}$ of $\bar{\alpha}\left(\mu, \bar{\Lambda}_{L}\right)$ is :

$$
\bar{\Lambda}_{L}=\left(\frac{\operatorname{det} \mathrm{N}}{\Lambda_{\mathrm{q}}^{\overline{\mathrm{b}}_{\mathrm{o}}}}\right)^{1 / 3 N_{c}}, \quad\left|\left\langle\bar{\Lambda}_{L}\right\rangle\right|=\Lambda_{Y M}
$$

Lowering the scale down to $\mu<\Lambda_{Y M}$ and integrating out all gauge degrees of freedom through the VY-procedure, one obtains the lowest energy Lagrangian:

$$
\bar{L}=\int d^{2} \theta d^{2} \bar{\theta}\left\{\frac{z_{M}}{\Lambda_{Q}^{2}} \operatorname{Tr}\left(\mathrm{M}^{\dagger} \mathrm{M}\right)+\operatorname{Tr} \sqrt{\mathrm{N}^{\dagger} \mathrm{N}}\right\}+
$$




$$
+\int d^{2} \theta\left\{\frac{1}{\Lambda_{Q}} \operatorname{Tr}(\mathrm{MN})+\left(\bar{N}_{c}-N_{F}\right)\left(\frac{\operatorname{det} \mathrm{N}}{\Lambda_{\mathrm{q}}^{\overline{\mathrm{b}}_{\mathrm{o}}}}\right)^{1 / N_{c}}+m_{Q} \operatorname{Tr} \mathrm{M}\right\} .
$$

Substituting $\Lambda_{q}$ from (4.4) and changing $\mathrm{N} \rightarrow(-\mathrm{N})$, its superpotential can be rewritten in a more convenient form :

$$
W=\frac{1}{\Lambda_{Q}} \operatorname{Tr}(-\mathrm{MN})+N_{c}\left(\frac{\operatorname{det} \mathrm{N}}{\Lambda_{\mathrm{Q}}^{\overline{\mathrm{b}}_{\mathrm{o}}}}\right)^{1 / N_{c}}+m_{Q} \operatorname{Tr} \mathrm{M} .
$$

Therefore, the masses of mions $\mathrm{M}$ and nions $\mathrm{N}$ are, see (5.2):

$$
\mu_{M} \sim \mu_{N} \sim\left(\frac{\bar{\mu}_{C}^{2}}{z_{M}}\right)^{1 / 2} \sim\left(\frac{m_{Q} \Lambda_{Q}}{z_{M}}\right)^{1 / 2}=\Lambda_{Q}\left(\frac{m_{Q}}{\Lambda_{Q}}\right)^{3 \bar{N}_{c} / 2 N_{F}} \ll \Lambda_{Y M} .
$$

On the whole, the mass spectrum looks here as follows: a) there is a large number of hadrons made of non-relativistic (and weakly confined, the string tension is $\sqrt{\sigma} \sim$ $\left.\Lambda_{Y M} \ll \bar{\mu}_{C}\right)$ dual quarks, with their dynamical constituent masses $\bar{\mu}_{C}=\left(m_{Q} \Lambda_{Q}\right)^{1 / 2} \ll$ $\Lambda_{Q}$, b) there is a large number of gluonia with their universal mass scale $\sim \Lambda_{Y M}$, c) the lightest are $\mathrm{N}_{\mathrm{F}}^{2}$ mions $\mathrm{M}$ and $\mathrm{N}_{\mathrm{F}}^{2}$ dual pions $\mathrm{N}$ (nions) with masses $\mu_{M} \sim \mu_{N} \sim$ $\Lambda_{Q}\left(m_{Q} / \Lambda_{Q}\right)^{3 \bar{N}_{c} / 2 N_{F}} \ll \Lambda_{Y M}$

Comparing the mass spectra of the direct and dual theories, it is seen that they are very different.

\section{Dual theory with $\mu_{q}=\mathcal{M}_{\mathrm{ch}}$. Conformal window}

Let us consider now this choice of parameters in (4.1). As will be shown below, this choice will result in a much more close similarity of the mass spectra of direct and dual theories.

But first, one obtains in this case from (4.4): $\left|\Lambda_{q}\right|=\left(\mathcal{M}_{\mathrm{ch}}^{N_{F}} / \Lambda_{Q}^{\mathrm{b}_{\mathrm{o}}}\right)^{1 / \overline{\mathrm{b}}_{\mathrm{o}}} \ll \Lambda_{Q}$, i.e. the scale parameter of the dual gauge coupling $\bar{\alpha}\left(\mu, \Lambda_{q}\right)$ is parametrically smaller than those of the direct one. Moreover, it is parametrically smaller than even $\mathcal{M}_{\mathrm{ch}}:\left(\left|\Lambda_{q}\right| / \mathcal{M}_{\mathrm{ch}}\right)=$ $\left(\mathcal{M}_{\mathrm{ch}} / \Lambda_{Q}\right)^{\mathrm{b}_{\mathrm{o}} / \overline{\mathrm{b}}_{\mathrm{o}}} \ll 1$. But this means that these two theories are clearly distinct in the perturbative interval $\mathcal{M}_{\mathrm{ch}}<\mu<\Lambda_{Q}$. Indeed, the direct theory entered already at $\mu<\Lambda_{Q}$ into the perturbative conformal regime, so that its coupling is frozen at the value $\alpha^{*}$ and does not run.

As for the dual theory, the most natural boundary condition at $\mu=\Lambda_{Q}$ is to take the scale factor $\Lambda_{f}$ of the Yukawa coupling $\Lambda_{f} \sim \Lambda_{q}$, this allows to consider self-consistently the dual theory as UV-free by itself (but nothing will change essentially at $\mu<\Lambda_{Q}$ also with $\Lambda_{f} \sim \Lambda_{Q}$, the Yukawa coupling will be $O(1)$ at $\mu \sim \Lambda_{Q}$ and will decrease then logarithmically with decreasing $\mu<\Lambda_{Q}$, the problem will be that the Yukawa coupling will grow with increasing $\mu$ at $\left.\mu>\Lambda_{Q}\right)$. With this choice, $a_{f}^{-1}\left(\mu=\Lambda_{Q}\right)=$ $2 \pi / N_{F} \alpha_{f}\left(\mu=\Lambda_{Q}\right) \sim(\bar{a})^{-1}\left(\mu=\Lambda_{Q}\right)=2 \pi / \bar{N}_{c} \bar{\alpha}\left(\mu=\Lambda_{Q}\right) \simeq \overline{\mathrm{b}}_{\mathrm{o}} \ln \left(\Lambda_{\mathrm{Q}} / \Lambda_{\mathrm{q}}\right) \gg 1$. Then, with decreasing $\mu<\Lambda_{Q}$, both couplings of the dual theory increase logarithmically but still remain $\ll 1$ at $\left|\Lambda_{q}\right| \ll \mathcal{M}_{\text {ch }}<\mu<\Lambda_{Q}$. So, the dual theory will be in the weak coupling logarithmic regime at $\mathcal{M}_{\mathrm{ch}} \ll \mu \ll \Lambda_{Q}$. Therefore, while correlators of the direct theory behave already in a power-like fashion, those of the dual one acquire only 
slow varying logarithmic renormalization factors. 10 Unfortunately, this is a price for a better similarity of both theories at lower scales $\mu<\mathcal{M}_{\mathrm{ch}}$. 11

The current mass of dual quarks is now $m_{q}=\mathcal{M}_{\mathrm{ch}}$, and it is much larger than the scale of their condensate: $|\langle q \bar{q}\rangle|^{1 / 2}=\left(m_{Q} \mathcal{M}_{\mathrm{ch}}\right)^{1 / 2}$. So, they can't be now in the collective coherent condensate phase, as their quantum fields are short ranged and will fluctuate independently locally. Therefore, they can be treated simply as heavy quarks (as their mass $\mathcal{M}_{\text {ch }}$ is much larger also than $\left|\Lambda_{q}\right|$ ). 12 Going to lower scales $\mu \ll \mathcal{M}_{\text {ch }}$, they can be integrated out directly as heavy particles. 13

What remains then, is the $S U\left(\bar{N}_{c}\right)$ Yang-Mills theory (plus the mions M) with the scale parameter $\bar{\Lambda}_{L}$ of its coupling $\bar{\alpha}(\mu)$ :

$\frac{2 \pi}{\bar{\alpha}\left(\mu, \bar{\Lambda}_{L}\right)}=3 \bar{N}_{c} \ln \frac{\mu}{\bar{\Lambda}_{L}}+\bar{N}_{c} \ln \frac{1}{\bar{g}^{2}\left(\mu,\left\langle\bar{\Lambda}_{L}\right\rangle\right)}, \quad-\bar{\Lambda}_{L}^{3}=\left(\operatorname{det} \mathrm{M} / \Lambda_{\mathrm{Q}}^{\mathrm{b}_{\mathrm{o}}}\right)^{1 / \overline{\mathrm{N}}_{\mathrm{c}}}, \quad\left|\left\langle\bar{\Lambda}_{\mathrm{L}}\right\rangle\right|=\Lambda_{\mathrm{YM}}(6.1)$

Therefore, at $\Lambda_{Y M} \ll \mu \ll \mathcal{M}_{\text {ch }}$, the effective dual Lagrangian takes the form (see the footnote 11):

$$
\bar{L}=\int d^{2} \theta d^{2} \bar{\theta}\left\{\frac{1}{\mathcal{M}_{\mathrm{ch}}^{2}} \operatorname{Tr}\left(\mathrm{M}^{\dagger} \mathrm{M}\right)\right\}+\int d^{2} \theta\left\{-\frac{2 \pi}{\bar{\alpha}\left(\mu, \bar{\Lambda}_{L}\right)} \bar{s}+m_{Q} \operatorname{TrM}\right\}
$$

Finally, at scales $\mu<\Lambda_{Y M}$, using the VY-procedure for integrating dual gluons, one obtains the lowest energy Lagrangian of mions :

$$
\begin{gathered}
\bar{L}=\int d^{2} \theta d^{2} \bar{\theta}\left\{\frac{1}{\mathcal{M}_{\mathrm{ch}}^{2}} \operatorname{Tr}\left(\mathrm{M}^{\dagger} \mathrm{M}\right)\right\}+ \\
+\int d^{2} \theta\left\{-\bar{N}_{c}\left(\frac{\operatorname{det} \mathrm{M}}{\Lambda_{Q}^{\mathrm{b}_{\mathrm{o}}}}\right)^{1 / \bar{N}_{c}}+m_{Q} \operatorname{TrM}\right\}, \mu \ll \Lambda_{Y M} .
\end{gathered}
$$

This describes the mions $\mathrm{M}$ with masses $\sim m_{Q}$, interacting weakly through the standard superpotential.

On the whole, let us compare the direct and dual theories in the case considered.-

a) As was pointed out above, they are clearly different in the region $\mathcal{M}_{\mathrm{ch}}<\mu<\Lambda_{Q}$.

b) There is a large number of colorless hadrons, the heavy mesons (quasi-stable, decaying into light pions or mions) and baryons (at least, those of lowest mass are stable) in both theories, made of heavy non-relativistic (and weakly confined, the string

\footnotetext{
10 Really, with so small value of $\left|\Lambda_{q}\right| \ll \mathcal{M}_{\text {ch }}$, the dual theory never enters the conformal regime, see below.

11 From now on, to simplify all expressions, in all those cases when the dual theory is in the weak coupling perturbative logarithmic regime, we will ignore the logarithmic renormalization factors $z_{q}$ and $z_{M}$ in calculations of mass spectra. In any case, because these non-leading effects from $z_{q} \neq 1$ and $z_{M} \neq 1$ are only logarithmic, taking them into account will not violate any power hierarchies and, besides, they are not of great importance for numerical values of masses.

12 Their non-zero vacuum condensate is now a pure quantum effect induced by the one-loop triangle diagram: $\left\langle\bar{q} q\left(\mu=\mathcal{M}_{\mathrm{ch}}\right)\right\rangle=\langle\bar{s}\rangle / \mathcal{M}_{\mathrm{ch}}$, where $\langle\bar{s}\rangle$ is the vacuum condensate of dual gluinos and $\mathcal{M}_{\mathrm{ch}} \gg$ $\Lambda_{Y M}$ is the large current mass of dual quarks. This realizes the Konishi anomaly.

13 Because the dual quarks are confined, this leaves behind a large number of mesons and baryons (with the mass scale $\sim \mathcal{M}_{\mathrm{ch}}$, the string tension is $\sqrt{\sigma} \sim \Lambda_{Y M} \ll \mathcal{M}_{\mathrm{ch}}$ ) made of weakly interacting non-relativistic heavy dual quarks with the current masses $\mathcal{M}_{\mathrm{ch}}$.
} 
tension is $\left.\sqrt{\sigma} \sim \Lambda_{Y M} \ll \mathcal{M}_{\mathrm{ch}}\right)$ constituents. In the direct theory these are the constituent quarks with the dynamically generated masses $\mu_{C}=\mathcal{M}_{\mathrm{ch}}$, while in the dual theory these are simply the dual quarks itself with the same (but now current) masses $\mathcal{M}_{\text {ch }}$. It seems that mesons are indistinguishable in both theories, but baryons are different because they know about a number of colors and their masses will be different: $M_{\text {baryon }}=N_{c} \mathcal{M}_{\text {ch }} \neq \bar{M}_{\text {baryon }}=\bar{N}_{c} \mathcal{M}_{\text {ch }}$.

c) The remaining light particles in both theories at $\Lambda_{Y M} \ll \mu \ll \mathcal{M}_{\text {ch }}$ are the gauge ones, with respectively $N_{c}$ and $\bar{N}_{c}$ colors, and pions (or mions). It is important that both Yang-Mills theories, direct and dual, are at weak couplings in this interval of scales, but have different numbers of colors. So, they are clearly different here. 14

d) There is a large number of (strongly coupled, quasi-stable due to decays into pions or mions) gluonia in both theories, all with masses determined the same scale $\Lambda_{Y M}$. So, it seems, they look indistinguishable.

e) Finally, there are $\mathrm{N}_{\mathrm{F}}^{2}$ of light pions (mions) with masses $\sim m_{Q}$ in both theories, weakly interacting at low energies $\mu \ll \Lambda_{Y M}$ through the same universal chiral superpotential. Nevertheless, as it is, the interactions of pions and mions with gluons at $\Lambda_{Y M} \ll \mu \ll \mathcal{M}_{\mathrm{ch}}$ are different in (3.10) and (6.2).

On the whole, it is seen that (with the logarithmic accuracy, see the footnote 11) the mass spectra look very similar in both theories in this case (but not completely). But in many other respects (see above) the direct and dual theories are clearly different.

\section{$7 \quad N_{c}<N_{F}<3 N_{c} / 2$}

There are two possible ways to interpret the meaning of the Seiberg dual theories at $N_{c}<N_{F}<3 N_{c} / 2$. -

a) The first variant is similar to those which is the only possibility in the conformal window $3 N_{c} / 2<N_{F}<3 N_{c}$. I.e., the description of all light degrees of freedom of the direct theory in terms of massless quarks $Q, \bar{Q}$ and gluons remains adequate in the interval of scales $\mu_{H} \ll \mu \leq \Lambda_{Q}$, where $\mu_{H} \ll \Lambda_{Q}$ is the highest physical mass scale due to $m_{Q} \neq 0$, and there are no massive particles with masses $\sim \Lambda_{Q}$ in the spectrum at $m_{Q} \ll \Lambda_{Q}$. In comparison with the conformal behavior, the difference is not qualitative but only quantitative: the strong coupling does not approach the constant value $\alpha^{*}$ at $\mu \ll \Lambda_{Q}$ but continues to grow. Nevertheless, the non-perturbative contributions are power suppressed until $\mu \gg \mu_{H}$, and one obtains the right answers for all Green functions by resummation of standard perturbative series with massless quarks and gluons. The dual theory is interpreted then as a possible alternative but equivalent (weak coupling) description. This variant can be thought of as some formal 'algebraic duality', i.e. something like 'the generalized change of variables'.

b) The second variant is qualitatively different (it is sometimes referred to as 'confinement without chiral symmetry breaking', i.e. due only to $\Lambda_{Q} \neq 0$ at $m_{Q} \rightarrow 0$ ). It implies that, unlike the variant 'a', the non-perturbative contributions become essential already at $\mu \sim \Lambda_{Q}$, resulting in a high scale confinement with the string tension $\sqrt{\sigma} \sim \Lambda_{Q}$ which binds direct quarks and gluons into colorless hadron states with masses $\sim \Lambda_{Q}$. This

14 Let us consider, for instance, the two-point correlators of the energy-momentum tensors in both theories. Because both gauge couplings are small and contributions from pion or mion interactions are already power suppressed at $\mu \ll \mathcal{M}_{\mathrm{ch}}$, these correlators are dominated by the lowest-order one-loop diagrams. The contributions of pions and mions are the same, but contributions of gauge particles are different, as $N_{c}^{2} \neq \bar{N}_{c}^{2}$. 
can be thought of, for instance, as follows. At $N_{F}$ close to $3 N_{c}$ the value of $a^{*}=N_{c} \alpha^{*} / 2 \pi$ is small. As $N_{F}$ decreases, $a^{*}$ increases and becomes $\simeq 1$ at $N_{F}$ close to $3 N_{c} / 2$. When $N_{F}<3 N_{c} / 2$, the coupling $a(\mu)$ exceeds some critical value $a^{(\text {crit })}=O(1)$ already at $\mu \sim \Lambda_{Q}$ and it is assumed that, for this reason, the theory is now in another phase. The strong non-perturbative confining gauge interactions begin to operate at the scale $\sim \Lambda_{Q}$, resulting in appearance of large number of colorless hadrons with masses $\sim \Lambda_{Q}$. So, the use of old massless quark and gluon fields for description of light degrees of freedom at $\mu \ll \Lambda_{Q}$ becomes completely inadequate. 15

Instead, the new (special solitonic ?) light degrees of freedom are formed at the scale $\sim \Lambda_{Q}$ as a result of these strong non-perturbative effects. These are the dual quarks and gluons and dual mesons $M$ (mions), with their sizes $\sim 1 / \Lambda_{Q}$ and the internal hardness scale $\sim \Lambda_{Q}$ (i.e. they appear as point-like at $\mu<\Lambda_{Q}$ ). These new light particles are described by fields of the dual theory. So, this variant ' $b$ ' can be thought of as 'the physical duality', in a sense that the dual theory is really the low energy description of the original theory at $\mu<\Lambda_{Q}$.

Now, we would like to present arguments against the variant 'b'. The above described scenario of 'confinement without chiral symmetry breaking' implies that, even at $m_{Q} \rightarrow 0$, there will be a large number of massive (with masses $\sim \Lambda_{Q}$ ) colorless hadrons $H_{n}$ in the spectrum, both non-chiral made of $\left(Q^{\dagger}, Q\right)$ or $\left(\bar{Q}^{\dagger}, \bar{Q}\right)$ quarks, and chiral made of $(\bar{Q}, Q)$ quarks, etc.

Let us consider, for instance, the action of the simplest colorless chiral superfield $\bar{Q} \bar{j} Q^{i}$ 16 on the vacuum state: $\bar{Q} \bar{j}^{i}|0\rangle$. This operator will excite from the vacuum not only, say, the massless one-mion state $\left|M_{\frac{i}{j}}\right\rangle$, but also many one-particle states of massive chiral hadrons $\left|\Psi_{n}\right\rangle$. Let $\Psi_{j}^{i}$ be the regular chiral superfield of anyone of such hadrons. Then in the effective Lagrangian describing theory at the scale $\mu \sim \Lambda_{Q}$ there should be a term in the superpotential which describes the nonzero mass $\sim \Lambda_{Q}$ of this chiral hadron. But the standard regular term $\Lambda_{Q} \operatorname{Tr}(\Psi \Psi)$ is not allowed as it breaks explicitly the chiral flavor $S U\left(N_{F}\right)_{L} \times S U\left(N_{F}\right)_{R}$ symmetry (and R-charge), and it seems impossible to write in the superpotential at $m_{Q} \rightarrow 0$ the appropriate regular mass terms for massive chiral hadron superfields with masses $\sim \Lambda_{Q}$. 17

\footnotetext{
${ }^{15}$ This is especially visible at $N_{F}=N_{c}+1$ where, for instance, the gauge degrees of freedom are not present at all amongst light ones in the dual theory.

${ }^{16}$ Or any other colorless spin zero or higher spin chiral superfield composed in some way from $Q^{i}, \bar{Q}_{\bar{j}}$ and the gauge field strength $W_{\alpha}$, for instance $\left(\bar{Q}-T^{a} Q^{i}\right) W_{\alpha}^{a}$, etc.

17 One can try to 'improve' situation multiplying the regular chiral superfield $\Psi_{j}^{i}$ by the chiral superfields $\left(\bar{Q}{ }_{j} Q^{i} / \Lambda_{Q}^{2}\right)^{-1}$ and $\left(\operatorname{det} \bar{Q}{ }_{j} Q^{i} / \Lambda_{Q}^{2 N_{F}}\right)^{1 / \Delta}$ to build up the term in the superpotential with appropriate quantum numbers, but all such terms are singular at $\left\langle 0\left|\bar{Q} \bar{j}^{i}\right| 0\right\rangle \rightarrow 0$, and so all this will not result in obtaining the genuine regular mass term for this hadron. Trying to use the dual quark fields $q$ and $\bar{q}$ together with $\Psi$ also does not help as $\langle\bar{q} q\rangle \rightarrow 0$ at $m_{Q} \rightarrow 0$.

One can also consider the variant of 'b' when the direct color is not confined.

From our point of view, this is the only realistic variant in the chiral limit $m_{Q}=0$. Because, at least in SQCD, the strong coupling $a\left(\mu \sim \Lambda_{Q}\right) \gtrsim 1$ does not mean really that the scale of confining forces is $\sim \Lambda_{Q}$ (in other words, that the string tension is $\sqrt{\sigma} \sim \Lambda_{Q}$ ). The underlying reason is that the role of the order parameter for the confinement plays not $\Lambda_{Q}$ by itself, but rather the scale of the gluino condensate, i.e. $\sqrt{\sigma} \sim \Lambda_{Y M}=\langle\lambda \lambda\rangle^{1 / 3}$. But $\langle\lambda \lambda\rangle \rightarrow 0$ at $m_{Q} \rightarrow 0$. So, there will be no confinement at all in the chiral limit $m_{Q}=0$, and the regimes at $m_{Q}=0$ and $N_{c}<N_{F}<3 N_{c}$ can be called more adequately as 'the pure perturbative massless regimes with neither confinement, nor chiral symmetry breaking', down to $\mu \rightarrow 0$. They are : conformal at $3 N_{c} / 2<N_{F}<3 N_{c}$, and strong coupling at $N_{c}<N_{F}<3 N_{c} / 2$, see (7.4) below).
}

Then, in the variant 'b', the absence of confinement at $m_{Q}=0$ implies that the individual quarks $Q^{i}$ 
In other words, the appearance in the spectrum of massive chiral flavored (and $\mathrm{R}$ charged) particles with masses $\sim \Lambda_{Q}$ at $m_{Q} \rightarrow 0$ seems impossible without the spontaneous breaking of $S U\left(N_{F}\right)_{L} \times S U\left(N_{F}\right)_{R}$ (and R-charge) symmetry.

If symmetry is broken spontaneously, there should be then the appropriate noninvariant (elementary or composite) chiral superfield(s) $\phi_{k}$ which condenses in the vacuum with the large value: $\left\langle 0\left|\phi_{k}\right| 0\right\rangle=\phi_{k}^{(o)} \sim \Lambda_{Q}$. This condensate can give then, in principle, the masses $\sim \Lambda_{Q}$ to chiral hadron superfields. But this basic condensate $\phi_{k}^{(o)}$ should figure then explicitly in the low energy Lagrangian, from which its numerical value in a chosen vacuum should be determined. The dual theory claims that it gives a right description at low energies. But no one so large chiral vacuum condensate $\phi_{k}^{(o)} \sim \Lambda_{Q}$ appear neither in the dual theory, nor in the direct one. We conclude that, indeed, the chiral flavor $S U\left(N_{F}\right)_{L} \times S U\left(N_{F}\right)_{R}$ and R-charge symmetries are not broken spontaneously at $m_{Q} \rightarrow 0$.

So, the above considerations imply that the scenario 'b' is incompatible with unbroken $S U\left(N_{F}\right)_{L} \times S U\left(N_{F}\right)_{R}$ (and R-charge) symmetries at $m_{Q} / \Lambda_{Q} \rightarrow 0$.

Therefore, we will consider below the scenario 'a' only in which the nonzero particle masses arise only due to breaking of the $S U\left(N_{F}\right)_{L} \times S U\left(N_{F}\right)_{R}$ and R-charge symmetries due to $m_{Q} \neq 0$, and these masses are all much smaller than $\Lambda_{Q}$ at $m_{Q} \ll \Lambda_{Q}$. Because in this variant the spectrum of light (i.e. with masses $\ll \Lambda_{Q}$ ) particles is known in both theories, direct and dual, it becomes possible, in addition to the 't Hooft triangles, to compare also the values of some special correlators in the perturbative range of energies where all particles can still be considered as being massless $\left(\mu_{H} \ll \mu \ll \Lambda_{Q}\right.$, where $\mu_{H}$ is the highest physical scale due to $\left.m_{Q} \neq 0\right)$. These are the two-point correlators of external conserved currents, say, the baryon and $S U\left(N_{F}\right)$ flavor currents, as these can be computed in the perturbation theory even in the strong coupling region. Really, it is more convenient to couple these conserved currents with the external vector fields and to consider the corresponding external $\beta_{\text {ext }}$-functions. Such $\beta_{\text {ext }}$-functions have the form (see e.g. [10]) :

$$
\frac{d}{d \ln \mu} \frac{2 \pi}{\alpha_{e x t}}=\sum_{i} T_{i}\left(1+\gamma_{i}\right)
$$

where the sum runs over all fields which can be considered as being massless at a given scale $\mu$, the unity in the brackets is due to one-loop contributions while the anomalous dimensions $\gamma_{i}$ of fields represent all higher-loop effects.

So, let us equate the values of such $\beta_{\text {ext }}$-functions in the direct and dual theories at scales $\mu_{H} \ll \mu \ll \Lambda_{Q}$. The light particles in the direct theory are the original quarks $Q, \bar{Q}$ and gluons, while in the dual theory these are the dual quarks $q, \bar{q}$ and dual gluons, and the mions M. For the baryon currents one obtains:

$$
N_{F} N_{c}\left(B_{Q}=1\right)^{2}\left(1+\gamma_{Q}\right)=N_{F} \bar{N}_{c}\left(B_{q}=\frac{N_{c}}{\bar{N}_{c}}\right)^{2}\left(1+\gamma_{q}\right)
$$

while for the $S U\left(N_{F}\right)_{L}$ (or $S U\left(N_{F}\right)_{R}$ ) flavor currents one obtains:

$$
N_{c}\left(1+\gamma_{Q}\right)=\bar{N}_{c}\left(1+\gamma_{q}\right)+N_{F}\left(1+\gamma_{M}\right)
$$

and $\bar{Q}_{\bar{j}}$ will be present in the spectrum and they will be massive, with masses $\sim \Lambda_{Q}$ (because there are no such light fields in the dual theory). And one will face the same problem that it is impossible to write in the superpotential the right regular mass term for these quarks. 
Here, the left-hand sides are from the direct theory while the right-hand sides are from the dual one, $\gamma_{Q}$ is the anomalous dimension of the quark $Q$, while $\gamma_{q}$ and $\gamma_{M}$ are the anomalous dimensions of the dual quark $q$ and the mion $\mathrm{M}$.

Now, at $\mu_{H} \ll \mu \ll \Lambda_{Q}$ the dual theory is IR-free and both its couplings are small in this range of energies, $\bar{a}(\mu) \ll 1, a_{f}(\mu) \ll 1$. So, $\gamma_{q}(\mu) \ll 1$ and $\gamma_{M}(\mu) \ll 1$ are both also logarithmically small at $\mu \ll \Lambda_{Q}$. It is seen then that (7.2) and (7.3) are incompatible with each other as they predict different values for the infrared limit of $\gamma_{Q}$. We conclude that both correlators can not be equal simultaneously in the direct and dual theories, and so these two theories are different. 18

Nevertheless, it is of interest to compare their mass spectra which will reveal itself at lower energies.

As for the direct theory, as was argued above, its qualitative properties don't differ much from those described before for the conformal window. The main quantitative difference is that the gauge coupling $\alpha(\mu)$ does not freeze at $\mu \ll \Lambda_{Q}$ but continues to grow (for instance, as in (7.4)), until $\mu$ reaches the scale of the dynamical chiral symmetry breaking, $\mu \sim \mu_{C}=\mathcal{M}_{\mathrm{ch}}$. But after crossing the threshold region $\mu_{2}=\mathcal{M}_{\mathrm{ch}} /$ (several $)<$ $\mu<\mu_{1}=$ (several) $\mathcal{M}_{\mathrm{ch}}$ the coupling also becomes logarithmically small, and the effective Lagrangian has the same form as in (3.2). Indeed, as was emphasized in section 3, this is independent of the explicit form of the quark perturbative renormalization factor $z_{Q}\left(\Lambda_{Q}, \mu_{1}\right)$ which enters the evolution of the coupling $\alpha^{-1}(\mu)$ in the region $\mu_{1}<\mu<\Lambda_{Q}$, because this last cancels in (3.8) independently of its explicit form. The only restriction is that the dynamical scenario has to be self-consistent. I.e., the constituent mass $\mu_{C}$ of quarks has to be larger than their perturbative pole mass, $\mu_{C}=\mathcal{M}_{\mathrm{ch}}>m_{Q}^{\text {pole }}$, so that it will stop the perturbative massless $R G$-evolution before this will be done by $m_{Q}^{\text {pole }}$. It is not difficult to check that this is fulfilled with $\gamma_{Q}=\left(2 N_{c}-N_{F}\right) /\left(N_{F}-N_{c}\right)$ from (7.2):

$$
\frac{m_{Q}^{\text {pole }}}{\Lambda_{Q}}=\frac{m_{Q}}{\Lambda_{Q}}\left(\frac{\Lambda_{Q}}{m_{Q}^{\text {pole }}}\right)^{\gamma_{Q}}=\left(\frac{m_{Q}}{\Lambda_{Q}}\right)^{\left(N_{F}-N_{c}\right) / N_{c}} \ll \frac{\mathcal{M}_{\mathrm{ch}}}{\Lambda_{Q}}=\left(\frac{m_{Q}}{\Lambda_{Q}}\right)^{\left(N_{F}-N_{c}\right) / 2 N_{c}}
$$

So, below the threshold region $\mu<\mu_{2}$, all equations and all qualitative properties of the direct theory described above for the conformal window remain the same also in the

18 Taking the IR value $\gamma_{Q} \rightarrow\left(N_{c} / \bar{N}_{c}-1\right)=\left(2 N_{c}-N_{F}\right) /\left(N_{F}-N_{c}\right)$ from (7.2) as a concrete example, and using the perturbative NSVZ $\beta$-function [5], one obtains the perturbative IR-behavior of the strong coupling $\alpha(\mu)$ :

$$
\begin{gathered}
\frac{d a(\mu)}{d \ln \mu} \equiv \beta(a)=-\frac{a^{2}}{1-a} \frac{\mathrm{b}_{\mathrm{o}}-\mathrm{N}_{\mathrm{F}} \gamma_{\mathrm{Q}}}{N_{c}}, \\
a(\mu) \equiv \frac{N_{c} \alpha(\mu)}{2 \pi}, \quad \mathrm{b}_{\mathrm{o}}=\left(3 \mathrm{~N}_{\mathrm{c}}-\mathrm{N}_{\mathrm{F}}\right), \quad \gamma_{\mathrm{Q}} \equiv \frac{\mathrm{d} \ln \mathrm{z}_{\mathrm{Q}}}{\mathrm{d} \ln \mu}, \quad \mathrm{z}_{\mathrm{Q}}\left(\Lambda_{\mathrm{Q}}, \mu\right)=\left(\frac{\mu}{\Lambda_{\mathrm{Q}}}\right)^{\gamma_{\mathrm{Q}}} \ll 1, \\
\gamma_{Q}=\frac{\left(2 N_{c}-N_{F}\right)}{\left(N_{F}-N_{c}\right)}, \quad a(\mu)=\left(\frac{\Lambda_{Q}}{\mu}\right)^{\nu} \gg 1, \quad \nu=\frac{3 N_{c}-2 N_{F}}{N_{F}-N_{c}}, \quad\left(\mu / \Lambda_{Q}\right) \ll 1 .
\end{gathered}
$$

In this case, the behavior of $a\left(\mu / \Lambda_{Q}\right)$ looks as follows. As $z=\mu / \Lambda_{Q}$ decreases from large values, $a(z)$ increases first in a standard way $\sim(1 / \ln z)$. At $z=z_{o} \sim 1 a(z)$ crosses unity. At this point $\gamma_{Q}$ crosses the value $b_{o} / N_{F}=\left(3 N_{c}-N_{f}\right) / N_{F}$. As a result, the $\beta$-function is smooth, it has neither pole nor zero at this point and remains negative all the way from the UV region $z \gg 1$ to the IR region $z \ll 1$, while $a(z)$ grows in the infrared region in a power-like fashion, see (7.4). On the other hand, it is not difficult to see that the IR-value of $\gamma_{Q}$ obtained from (7.3) with $\gamma_{q} \rightarrow 0, \gamma_{M} \rightarrow 0$ is incompatible with the NSVZ $\beta$-function. 
region $N_{c}<N_{F}<3 N_{c} / 2$.

As for the dual theory, we also consider here two variants for the scale parameter $\mu_{q}$ in (4.1): a) $\mu_{q}=\Lambda_{Q}$, and b) $\mu_{q}=\mathcal{M}_{\mathrm{ch}}$.

a) $\mu_{q}^{\prime} \sim \mu_{q}=\Lambda_{Q}$

In this case the scale parameter $\Lambda_{q}$ of the dual gauge coupling $\bar{\alpha}(\mu)$ is : $\left|\Lambda_{q}\right| \sim \Lambda_{f} \sim$ $\Lambda_{Q}$, see (4.4), both couplings $\bar{a}(\mu)$ and $a_{f}(\mu)$ are $\lesssim 1$ at $\mu=\Lambda_{Q}$ and both decrease logarithmically when $\mu$ is going down from $\mu \sim \Lambda_{Q}$ to $\mu_{H} \sim \mathcal{M}_{\text {ch }}^{2} / \Lambda_{Q} \ll \Lambda_{Q}$.

In the case considered, the current mass of dual quarks is (see the footnote 11):

$$
m_{q}=\langle\mathrm{M}\rangle / \mu_{q}=\mathcal{M}_{\mathrm{ch}}^{2} / \Lambda_{Q}, \quad m_{q} \gg|\langle\bar{q} q\rangle|^{1 / 2}=\left(m_{Q} \Lambda_{Q}\right)^{1 / 2},
$$

i.e. it is much larger than the scale of their condensate, so that the dual theory is here in the same HQ (heavy quark) phase as it was in section 6. Therefore, at lower scales all quarks can simply be integrated out as heavy (and weakly confined, the string tension is $\left.\sqrt{\sigma} \sim \Lambda_{Y M} \ll \mathcal{M}_{\mathrm{ch}}^{2} / \Lambda_{Q}\right)$ particles, leaving behind a large number of hadrons with masses $\sim \mathcal{M}_{\mathrm{ch}}^{2} / \Lambda_{Q}$ composed of non-relativistic dual quarks. After this, one obtains the effective Lagrangian in the form:

$$
\begin{gathered}
\bar{L}=\left\{\frac{1}{\Lambda_{Q}^{2}} \operatorname{Tr}\left(\mathrm{M}^{\dagger} \mathrm{M}\right)\right\}_{D}+\left\{-3 \bar{N}_{c} \bar{s}\left[\ln \frac{\mu}{\bar{\Lambda}_{L}}+\ln \frac{1}{\bar{g}^{2}\left(\mu / \Lambda_{Y M}\right)}\right]+m_{Q} \operatorname{Tr} \mathrm{M}\right\}_{F}, \\
\bar{\Lambda}_{L}^{3}=-\left(\operatorname{det} \mathrm{M} / \Lambda_{Q}^{\mathrm{b}_{\mathrm{o}}}\right)^{1 / \bar{N}_{c}}, \quad\left|\left\langle\bar{\Lambda}_{L}\right\rangle\right|=\Lambda_{Y M}, \quad \Lambda_{Y M} \ll \mu \ll \mathcal{M}_{\mathrm{ch}}^{2} / \Lambda_{Q} .
\end{gathered}
$$

Going down in energy and integrating out all gluonia (with masses $\sim \Lambda_{Y M}$ ) through the VY- procedure, one obtains finally:

$$
\bar{L}=\left\{\frac{1}{\Lambda_{Q}^{2}} \operatorname{Tr}\left(\mathrm{M}^{\dagger} \mathrm{M}\right)\right\}_{D}+\left\{-\bar{N}_{c}\left(\frac{\operatorname{det} \mathrm{M}}{\Lambda_{Q}^{\mathrm{b}_{o}}}\right)^{1 / \bar{N}_{c}}+m_{Q} \operatorname{Tr} \mathrm{M}\right\}_{F}, \mu \ll \Lambda_{Y M} .
$$

This describes the mions $\mathrm{M}$ with the masses:

$$
\mu_{M} \sim m_{Q}\left(\frac{\Lambda_{Q}^{2}}{\mathcal{M}_{\mathrm{ch}}^{2}}\right) \sim m_{Q}\left(\frac{\Lambda_{Q}}{m_{Q}}\right)^{\bar{N}_{c} / N_{c}}, \quad m_{Q} \ll \mu_{M} \ll \Lambda_{Y M},
$$

interacting weakly through the standard superpotential.

So, comparing the mass spectra of the direct and dual theories one sees that they are very different.

b) $\mu_{q}=\mathcal{M}_{\mathrm{ch}}$. $(4.4)$ :

With $\Lambda_{f} \sim\left|\Lambda_{q}\right|$, both dual scale factors become very large with this choice of $\mu_{q}$, see

$$
\left|\Lambda_{q}\right|=\left(\frac{\Lambda_{Q}^{\mathrm{b}_{\mathrm{o}}}}{\mathcal{M}_{\mathrm{ch}}^{N_{F}}}\right)^{\left(-1 / \overline{\mathrm{b}_{\mathrm{o}}}\right)} \gg \Lambda_{Q}
$$


But we can ignore the high energy region $\mu>\left|\Lambda_{q}\right|$ where the dual theory is strongly coupled, and to start directly with $\mu \lesssim \Lambda_{Q} \ll\left|\Lambda_{q}\right|$, where both couplings are already logarithmically small : $2 \pi / \alpha_{f}\left(\mu=\Lambda_{Q}\right) \sim 2 \pi / \bar{\alpha}\left(\mu=\Lambda_{Q}\right) \simeq \overline{\mathrm{b}}_{\mathrm{o}} \ln \left(\left|\Lambda_{\mathrm{q}}\right| / \Lambda_{Q}\right) \gg 1$, and both continue to decrease logarithmically with decreasing $\mu$ at $\mathcal{M}_{\mathrm{ch}}<\mu<\left|\Lambda_{Q}\right|$. The region $\mathcal{M}_{\text {ch }}<\mu<\Lambda_{Q}$ was discussed above, see (7.2),(7.3). So, let us consider now $\mu<\mathcal{M}_{\text {ch }}$.

The regime in this case b) is qualitatively the same as in the case a) above (see also the footnote 11), i.e. there are now even heavier dual quarks with the current mass $m_{q}=\mathcal{M}_{\mathrm{ch}}$ (and even smaller condensate), the intermediate mass gluonia and smallest mass mions M. And (7.6), (7.7) remain essentially the same, only the factor $1 / \Lambda_{Q}^{2}$ in the meson Kahler term is replaced now by $1 / \mathcal{M}_{\mathrm{ch}}^{2}$. Due to this, the masses $\mu_{M}$ of mions are now:

$$
m_{q} \sim \mathcal{M}_{\mathrm{ch}} \gg M_{\mathrm{gl}} \sim \Lambda_{Y M} \gg \mu_{M} \sim m_{Q} .
$$

So, in this case the mass spectra of the direct and dual theories (with the logarithmic accuracy) are much more similar, as it was in section 6 in the conformal window. But all differences (at scales $\mu<\mathcal{M}_{\mathrm{ch}}$ ) described in section 6 also remain.

c) $\quad N_{F}=N_{c}+1$.

As for the direct theory, this point is not special and all equations and results described before remain without changes. 19

But this point is somewhat special for the dual theory because its field content consists in this case of light mesons $\mathrm{M}_{\overline{\mathrm{j}}}^{\mathrm{i}}$ and baryons $\mathrm{B}_{\mathrm{i}}, \overline{\mathrm{B}}^{\overline{\mathrm{j}}}$ only [1].

The dual Lagrangian at $\mu<\Lambda_{Q}$ is supposed to have the form [1] :

$$
\begin{gathered}
\bar{L}=\int d^{2} \theta d^{2} \bar{\theta}\left\{\frac{\mathrm{M}^{\dagger} \mathrm{M}}{\mu_{\mathrm{M}}^{2}}+\frac{\mathrm{B}^{\dagger} \mathrm{B}+\overline{\mathrm{B}}^{\dagger} \overline{\mathrm{B}}}{\mu_{\mathrm{B}}^{2\left(\mathrm{~N}_{\mathrm{c}}-1\right)}}\right\}+ \\
+\int d^{2} \theta\left\{\frac{\operatorname{Tr}(\overline{\mathrm{B}} \mathrm{MB})-\operatorname{det} \mathrm{M}}{\Lambda_{\mathrm{Q}}^{\mathrm{b}_{\mathrm{o}}}}+\mathrm{m}_{\mathrm{Q}} \operatorname{Tr} \mathrm{M}\right\}, \quad \mu \ll \Lambda_{\mathrm{Q}} .
\end{gathered}
$$

Here: the scale factors $\mu_{M}$ and $\mu_{B}$ in the Kahler terms are due to non-canonical dimensions of meson and baryon fields $\left(M \rightarrow \bar{Q} Q, B \rightarrow Q^{N_{c}}\right)$.

As for the interval of energies above the highest physical scale $\mu_{H}, \mu_{H} \ll \mu \ll \Lambda_{Q}$, the equations $(7.2,7.3)$ still hold in this case, with substitution: $\bar{N}_{c}=1, \gamma_{q} \rightarrow \gamma_{B}$, and $\gamma_{M}, \gamma_{B} \rightarrow 0$. So, they remain incompatible.

At lower energies, the meson and baryon masses can be obtained directly from the Lagrangian (7.11):

$$
M_{M} \sim m_{Q}\left(\frac{\mu_{M}}{\mathcal{M}_{\mathrm{ch}}}\right)^{2}, \quad M_{B}=M_{\bar{B}} \sim \frac{\mathcal{M}_{\mathrm{ch}}^{2} \mu_{B}^{2\left(N_{c}-1\right)}}{\Lambda_{Q}^{\mathrm{b}_{\mathrm{o}}}} .
$$

So, at $\mu_{M} \sim \mu_{B} \sim \Lambda_{Q}: M_{M} \sim \Lambda_{Q}\left(m_{Q} / \Lambda_{Q}\right)^{\left(N_{c}-1\right) / N_{c}}, B_{B} \sim \Lambda_{Q}\left(m_{Q} / \Lambda_{Q}\right)^{1 / N_{C}}$. Let us recall (see above) that the mass spectrum of the direct theory consists here also of a large number of flavored hadrons with the mass scale $\sim \mathcal{M}_{\mathrm{ch}} \sim \Lambda_{Q}\left(m_{Q} / \Lambda_{Q}\right)^{1 /\left(2 N_{c}\right)}$, a large number of gluonia with masses $M_{\mathrm{gl}} \sim \Lambda_{Y M} \sim \Lambda_{Q}\left(m_{Q} / \Lambda_{Q}\right)^{\left(N_{c}+1\right) / 3 N_{c}}$, and $N_{F}^{2}$ light pions with masses $\sim m_{Q}$.

19 Really special is the point $N_{F}=N_{c}$, as $\mathcal{M}_{\mathrm{ch}}=\Lambda_{Q}$ in this case, even in the chiral limit $m_{Q} \rightarrow 0$, see (2.7). We do not consider this case here. 


\section{$8 \quad N_{F}>3 N_{c}$}

For completeness, let us also consider this region.

As for the direct theory, it is IR-free in this region $\left(\mathrm{b}_{\mathrm{o}}<0\right)$ at $m_{Q}^{\text {pole }}<\mu<\Lambda_{Q}$. So, in a sense, it is very "simple" at $\mu \gg \Lambda_{Y M}$ (but at the price that it is now, at best, strongly coupled in the UV-region $\mu \gg \Lambda_{Q}$ and, at worst, can't be defined self-consistently in UV by itself and needs the UV completion).

The current quark mass $m_{Q}=m_{Q}\left(\mu=\Lambda_{Q}\right) \ll \Lambda_{Q}$ is much larger now than the scale of its chiral condensate $\mathcal{M}_{\text {ch }} \ll m_{Q}$, see (2.7), and this power hierarchy persists at lower energies as the RG - evolution here is only logarithmic at $\Lambda_{Y M} \ll \mu<\Lambda_{Q}$. Therefore, the direct theory is at $N_{F}>3 N_{c}$ in the HQ (heavy quark) phase, so that there is a standard weak coupling slow logarithmic evolution in the region $m_{Q}^{\text {pole }} \ll \mu \ll \Lambda_{Q}, m_{Q}^{\text {pole }} \equiv m_{Q}(\mu=$ $\left.m_{Q}^{\text {pole }}\right)=z_{Q}^{-1}\left(\Lambda_{Q}, \mu=m_{Q}^{\text {pole }}\right) m_{Q} \gg m_{Q}$, where $z_{Q}\left(\Lambda_{Q}, \mu=m_{Q}^{\text {pole }}\right) \ll 1$ is the standard perturbative logarithmic renormalization factor of massless quarks, and the highest physical scale is now : $\mu_{H}=m_{Q}^{\text {pole }} \gg \Lambda_{\mathrm{YM}} \gg \mathcal{M}_{\mathrm{ch}}$. At $\mu \ll m_{Q}^{\text {pole }}$ all quarks can be integrated out as heavy (and weakly confined, the string tension is $\sqrt{\sigma} \sim \Lambda_{Y M} \ll m_{Q}^{\text {pole }}$, their vacuum condensate $\left\langle\bar{Q} Q\left(\mu=m_{Q}^{\text {pole }}\right)\right\rangle=\langle S\rangle / m_{Q}^{\text {pole }}$ is due to a simple quantum one-loop contribution ) non-relativistic particles, leaving behind a large number of mesons and baryons made of these non-relativistic quarks, with masses : $\mathrm{M}_{\text {meson }} \sim \mathrm{m}_{\mathrm{Q}}^{\text {pole }}, \mathrm{M}_{\text {baryon }} \sim \mathrm{N}_{\mathrm{c}} \mathrm{m}_{\mathrm{Q}}^{\text {pole }}$. Evidently, there are no additional lighter pions now.

Using $(2.2,2.3)$ to match couplings at $\mu=m_{Q}^{\text {pole }}$, one obtains at lower energies $\mu<m_{Q}^{\text {pole }}$ the Yang-Mills Lagrangian with the scale factor of its gauge coupling $\Lambda_{Y M}=$ $\left(\Lambda_{Q}^{\mathrm{b}_{\mathrm{o}}} m_{Q}^{N_{F}}\right)^{1 / 3 N_{c}} \ll m_{Q}$, so that this Yang-Mills theory is in the weak coupling regime at $\Lambda_{Y M} \ll \mu<m_{Q}^{\text {pole }}$. Finally, it describes strongly coupled gluonia with masses $M_{\mathrm{gl}} \sim \Lambda_{Y M} \ll m_{Q}^{\text {pole }}$, and these are the lightest particles in this case. So, this is the end of this short story in the direct theory.

As for the dual theory, as before, its mass spectrum depends on the value of $\mu_{q}$.

\section{a. Dual theory with $\mu_{q}^{\prime} \sim \mu_{q}=\Lambda_{Q}$}

$\Lambda_{f} \sim\left|\Lambda_{q}\right| \sim \Lambda_{Q}$, see (4.4), but there are no particles with masses $\sim \Lambda_{Q}$, similarly as it was for the direct theory in section 7 . The dual theory is taken as UV-free in this case and it enters the strong coupling perturbative regime at $\mu_{H}<\mu<\Lambda_{Q}$. For definiteness, let us use the values of the dual quark and mion anomalous dimensions from (7.2), (7.3) with $\gamma_{Q} \rightarrow 0$ at $\mu \ll \Lambda_{Q}$ :

$$
\gamma_{q}=\frac{\bar{N}_{c}}{N_{c}}-1, \quad \gamma_{M}=-\frac{\bar{N}_{c}}{N_{c}}
$$

Now, it is seen that the dynamical constituent mass of dual quarks $\bar{\mu}_{C}$ is parametrically larger here than their pole mass $m_{q}^{\text {pole }}$ :

$$
\bar{\mu}_{C}=\left(m_{Q} \Lambda_{Q}\right)^{1 / 2} \gg m_{q}^{\text {pole }}, \quad m_{q}^{\text {pole }}=\frac{\mathcal{M}_{\mathrm{ch}}^{2}}{\Lambda_{Q}}\left(\frac{\Lambda_{Q}}{m_{q}^{\text {pole }}}\right)^{\gamma_{q}}=\Lambda_{Q}\left(\frac{\mathcal{M}_{\mathrm{ch}}^{2}}{\Lambda_{Q}^{2}}\right)^{1 /\left(1+\gamma_{q}\right)}=m_{Q} .
$$

So, $\mu_{H}=\bar{\mu}_{C}$ and the dual quarks are in the (dual) DC - phase. The Lagrangian has the same form (5.1), all equations (5.3-5.6) remain the same and, instead of (5.7), the 
masses of mions and nions look now as:

$$
\frac{\mu_{M}}{\Lambda_{Q}} \sim \frac{\mu_{N}}{\Lambda_{Q}} \sim\left(\frac{\bar{\mu}_{C}^{2}}{z_{M} \Lambda_{Q}^{2}}\right)^{1 / 2}=\left(\frac{m_{Q}}{\Lambda_{Q}}\right)^{\left(N_{F}+N_{c}\right) / 4 N_{c}}, \quad z_{M}=\left(\frac{\bar{\mu}_{C}}{\Lambda_{Q}}\right)^{\gamma_{M}} \gg 1 .
$$

On the whole, the mass spectrum of the dual theory includes in this case: a) a large number of flavored hadrons with their mass scale $\sim \bar{\mu}_{C}$, made of dual quarks with the constituent masses $\bar{\mu}_{C}=\left(m_{Q} \Lambda_{Q}\right)^{1 / 2} \ll \Lambda_{Q}$, b) $\mathrm{N}_{F}^{2}$ mions and $\mathrm{N}_{F}^{2}$ nions with masses $\mu_{M} \sim \mu_{N} \sim \Lambda_{Q}\left(m_{Q} / \Lambda_{Q}\right)^{\left(N_{F}+N_{c}\right) / 4 N_{c}} \ll \bar{\mu}_{C}$, c) a large number of gluonia with the mass scale $\sim \Lambda_{Y M} \ll \mu_{M} \sim \mu_{N}$.

\section{b. Dual theory with $\mu_{q}=\mathcal{M}_{\mathrm{ch}}$.}

With the choice $\left|\Lambda_{q}\right| \sim \Lambda_{f}$, both are very small in this case, see (4.4). The dual theory is also taken as UV-free at $\mu>\left|\Lambda_{q}\right|$ in this case, and it will also enter the strong coupling perturbative regime at $\mu_{H}<\mu<\left|\Lambda_{q}\right|$. The boundary conditions for the dual gauge coupling $\bar{a}=\bar{N}_{c} \bar{\alpha} / 2 \pi$ and the Yukawa coupling $a_{f}=N_{F} f^{2} / 2 \pi$ at $\mu=\Lambda_{Q}$ look as: $\bar{a}\left(\mu=\Lambda_{Q}\right) \sim a_{f}\left(\mu=\Lambda_{Q}\right) \sim 1 / \ln \left(\Lambda_{Q} /\left|\Lambda_{q}\right|\right) \ll 1$. In the perturbative regions: $\Lambda_{Y M} \ll$ $\mu \ll \Lambda_{Q}$ for the direct theory and $\Lambda_{Y M} \ll\left|\Lambda_{q}\right| \ll \mu \ll \Lambda_{Q}$ for the dual one, both theories are now in the weak coupling logarithmic regime. The direct theory - because it is IR-free at $\left|\Lambda_{q}\right| \ll m_{Q}^{\text {pole }} \ll \mu \ll \Lambda_{Q}$, while its coupling $a(\mu)$ increases logarithmically at $\Lambda_{Y M} \ll \mu \ll m_{Q}^{\text {pole }}$ but is still small. The dual theory - because $\left|\Lambda_{q}\right| \sim \Lambda_{f}$ are so small, both its couplings $\bar{a}(\mu)$ and $a_{f}(\mu)$ increase logarithmically with decreasing $\mu<\Lambda_{Q}$ but still remain small at $\mu \gg\left|\Lambda_{q}\right|$. So, at $m_{Q}^{\text {pole }} \ll \mu \ll \Lambda_{Q}$ the direct and dual theories are both in the weak coupling logarithmic perturbative massless regime, the equations $(7.2,7.3)$ can be used with all $\gamma_{Q}, \gamma_{q}, \gamma_{M} \ll 1$ now, and they are incompatible.

At $\mu \ll\left|\Lambda_{q}\right|$ the dual theory is in the strong coupling regime $\bar{a}(\mu) \gg 1, a_{f}(\mu) \gg 1$, and we use for the anomalous dimensions $\gamma_{q}$ and $\gamma_{M}$ the values (8.1).

The hierarchies in the dual theory at $\mu=\Lambda_{Q} \gg\left|\Lambda_{q}\right|$ look as:

$$
m_{q}=\mathcal{M}_{\mathrm{ch}} \ll \bar{\mu}_{C}=|\langle\bar{q} q\rangle|^{1 / 2}=\left(m_{Q} \mathcal{M}_{\mathrm{ch}}\right)^{1 / 2} \ll\left|\Lambda_{q}\right|, \quad \frac{\left|\Lambda_{q}\right|}{\Lambda_{Q}}=\left(\frac{\mathcal{M}_{\mathrm{ch}}}{\Lambda_{Q}}\right)^{N_{F} / \overline{\mathrm{b}}_{\mathrm{o}}} \ll 1,
$$

where $m_{q}$ is the current quark mass and $\bar{\mu}_{C}$ is its (possible) constituent mass. The evolution in the interval $\left|\Lambda_{q}\right|<\mu<\Lambda_{Q}$ is only logarithmic ( all logarithmic effects are neglected in what follows) and the hierarchies at $\mu \sim\left|\Lambda_{q}\right|$ remain the same. The dual quarks will be in the DC - phase with the constituent mass $\bar{\mu}_{C}=|\langle\bar{q} q\rangle|^{1 / 2}=\left(m_{Q} \mathcal{M}_{\mathrm{ch}}\right)^{1 / 2} \ll\left|\Lambda_{q}\right|$ if $\bar{\mu}_{C} \gg m_{q}^{\text {pole }}$, where $m_{q}^{\text {pole }}$ is the pole mass of dual quarks. This is fulfilled, see (8.1):

$$
\frac{m_{q}^{\text {pole }}}{\left|\Lambda_{q}\right|}=\left(\frac{m_{q}}{\left|\Lambda_{q}\right|}\right)^{\frac{1}{1+\gamma_{q}}}=\left(\frac{\bar{\mu}_{C}}{\left|\Lambda_{q}\right|}\right)^{2} \ll \frac{\bar{\mu}_{C}}{\left|\Lambda_{q}\right|} \ll 1, \quad \Lambda_{Y M} \ll \bar{\mu}_{C} \ll\left|\Lambda_{q}\right| .
$$

So, the Lagrangian of mions and nions will have the form (5.5),(5.6), with the only replacement $\Lambda_{Q} \rightarrow \mathcal{M}_{\text {ch }}$ in the mion Kahler term and in the first term of the superpotential. So, instead of (8.2), the masses of mions and nions (with the logarithmic accuracy) are now :

$$
\frac{\mu_{M}}{\left|\Lambda_{q}\right|} \sim \frac{\mu_{N}}{\left|\Lambda_{q}\right|} \sim\left(\frac{\bar{\mu}_{C}^{2}}{z_{M}\left|\Lambda_{q}\right|^{2}}\right)^{1 / 2}=\left(\frac{\bar{\mu}_{C}}{\left|\Lambda_{q}\right|}\right)^{\left(N_{F}+N_{c}\right) / 2 N_{c}} \quad, \quad z_{M}=\left(\frac{\bar{\mu}_{C}}{\left|\Lambda_{q}\right|}\right)^{\gamma_{M}} \gg 1 .
$$


Let us finish this section with a short discussion of a possible behavior of the direct theory in the case $m_{Q} \gg \Lambda_{Q}$. We have to start then from the UV-region $\mu=M_{o}$, supposing that this theory is considered as the effective low energy theory with the UV cutoff $M_{o}$.

Let us use (2.7) for $N_{F}>3 N_{c}$. It is seen that the hierarchy of the standard scale parameters at $\mu=\Lambda_{Q}$ and $N_{F}>3 N_{c}, m_{Q} \gg \Lambda_{Q}$ remains the same as it was at $N_{F}<3 N_{c}$ and $m_{Q} \ll \Lambda_{Q}$, i.e. : $\mathcal{M}_{\mathrm{ch}} \gg \Lambda_{Y M} \gg m_{Q}$. But what is really the highest physical scale $\mu_{H}$ depends on a competition between $\mathcal{M}_{\mathrm{ch}}$ and the quark pole mass $m_{Q}^{\text {pole }}$. The value of this last depends on the value of the quark anomalous dimension $\gamma_{Q}$. If $\mathcal{M}_{\mathrm{ch}}>m_{Q}^{\text {pole }}$, the theory will be in the DC - phase, while at $m_{Q}^{\text {pole }}>\mathcal{M}_{\text {ch }}$ it will be in the HQ (heavy quark) phase.

For definiteness, let us use the same value of $\gamma_{Q}$ as in (7.2) with $\gamma_{q} \rightarrow 0$ :

$$
\gamma_{Q}=\left(2 N_{c}-N_{F}\right) /\left(N_{F}-N_{c}\right)<0 \quad \text { at } \quad N_{F}>3 N_{c}
$$

Then

$$
\frac{m_{Q}^{\text {pole }}}{\Lambda_{Q}}=\left(\frac{m_{Q}}{\Lambda_{Q}}\right)^{\frac{1}{1+\gamma_{Q}}}=\left(\frac{m_{Q}}{\Lambda_{Q}}\right)^{\frac{N_{F}-N_{c}}{N_{c}}} \gg \frac{\mathcal{M}_{\mathrm{ch}}}{\Lambda_{Q}}=\left(\frac{m_{Q}}{\Lambda_{Q}}\right)^{\frac{N_{F}-N_{c}}{2 N_{c}}}, \quad \frac{m_{Q}}{\Lambda_{Q}} \gg 1 .
$$

Therefore, with this value of $\gamma_{Q}$, when going from high UV $\mu=M_{o} \gg m_{Q}^{\text {pole }}$ down to lower energies, the highest physical scale encountered is $\mu_{H}=m_{Q}^{\text {pole }}$. The quarks will be in the HQ (heavy quark) phase.

After integrating out all quarks as heavy ones, one remains with the pure Yang-Mills theory, but now in the strong coupling regime, $a_{-}=N_{c} \alpha\left(\mu=m_{Q}^{\text {pole }}\right) / 2 \pi \gg 1$. So, the matching of couplings at $\mu=m_{Q}^{\text {pole }}$ looks now as follows. The coupling of the higher energy theory is, see (7.4) :

$$
a_{+}=\left(\frac{m_{Q}^{\text {pole }}}{\Lambda_{Q}}\right)^{-\nu=\left(\frac{2 N_{F}-3 N_{c}}{N_{F}-N_{c}}\right)}=\left(\frac{m_{Q}}{\Lambda_{Q}}\right)^{\frac{2 N_{F}-3 N_{c}}{N_{c}}} \gg 1 .
$$

It follows from the perturbative NSVZ $\beta$ - function [5] that the coupling of the lower energy Yang-Mills theory in the strong coupling regime is : $a_{-}\left(\mu \gg \lambda_{Y M}\right)=\left(\mu / \lambda_{Y M}\right)^{3}$. So,

$$
a_{-}=\left(\frac{m_{Q}^{\text {pole }}}{\lambda_{Y M}}\right)^{3}=a_{+} \rightarrow \lambda_{Y M}=\left(\Lambda_{Q}^{\mathrm{b}_{\mathrm{o}} \operatorname{det} m_{Q}}\right)^{1 / 3 N_{c}}=\Lambda_{Y M} \gg \Lambda_{Q} .
$$

We have now the Yang-Mills theory in the strong coupling perturbative regime at $\Lambda_{Y M} \ll \mu<m_{Q}^{\text {pole }}$, with its coupling decreasing with $\mu$ as $a(\mu)=\left(\mu / \Lambda_{Y M}\right)^{3}$ until it becomes $O(1)$ at $\mu \sim \Lambda_{Y M}$, and here the non-perturbative effects come into a game. So, integrating at $\mu<\Lambda_{Y M}$ all gauge degrees of freedom, except for the one whole field $S \sim W_{\alpha}^{2}$, and using the VY - form for the superpotential of $S$ [6] one obtains the right value of the gluino condensate, $\langle S\rangle=\Lambda_{Y M}^{3}$ (and a large number of gluonia with the mass scale $\left.\sim \Lambda_{Y M}\right)$.

On the whole, the mass spectrum includes only two mass scales in this case: a large number of heavy flavored quarkonia with the mass scale $\sim m_{Q}^{\text {pole }} \gg \Lambda_{Y M}$, and a large number of gluonia with the universal mass scale $\sim \Lambda_{Y M} \gg \Lambda_{Q}$. 


\section{Conclusions}

As was described above, within the dynamical scenario considered in this paper, the direct SQCD theory is in the DC (diquark-condensate) phase at $N_{c}<N_{F}<3 N_{c}$. In this case, its properties and the mass spectrum were described and compared with those of the dual theory. It was shown that the direct and dual theories are different, in general. The only region where no difference was found up to now, is the case when both theories are in the perturbative superconformal regime (see above). All this can be of significance in a wider aspect, - as a hint that many of various dualities considered in the literature can be strictly valid, at best, also in the superconformal regime only.

We will not repeat here in detail the above described results. Rather, let us compare the gross features of SQCD and ordinary QCD. The above described properties of SQCD at $N_{c}<N_{F}<3 N_{c}$ resemble, in many respects, those of QCD. 20 I.e., there is simultaneously confinement and chiral flavor symmetry breaking, with formation of heavy constituent quarks and light pions. Besides, in both theories there is a large number of (quasi) stable heavy quarkonia and gluonia. The main difference is in the parametrical dependence of different observable masses in the spectrum on the fundamental parameters of Lagrangians: $\Lambda_{Q}$ and the current quark masses $m_{Q}=m_{Q}\left(\mu=\Lambda_{Q}\right)$, when $m_{Q} \ll \Lambda_{Q}$.

a) The scale of the chiral symmetry breaking $\Lambda_{\mathrm{ch}}$ (and so the masses of constituent quarks) is $\Lambda_{\mathrm{ch}}^{Q C D} \sim \Lambda_{Q}$ in QCD, while it is parametrically smaller in SQCD: $\Lambda_{\mathrm{ch}}^{S Q C D} \sim$ $\mathcal{M}_{\mathrm{ch}}=\left(\Lambda_{Q}^{\mathrm{b}_{\mathrm{o}}} m_{Q}^{\bar{N}_{c}}\right)^{1 / 2 N_{c}} \ll \Lambda_{Q}$.

b) The confinement scale (i.e. the string tension $\sqrt{\sigma}$ ) is $\Lambda_{\text {conf }}^{Q C D}=\left(\sigma_{Q C D}\right)^{1 / 2} \sim \Lambda_{Q} \sim$ $\Lambda_{\mathrm{ch}}^{Q C D}$ in QCD, while it is parametrically smaller than even $\Lambda_{\mathrm{ch}}^{S Q C D}$ in SQCD: $\Lambda_{\text {conf }}^{S Q C D}=$ $\left(\sigma_{S Q C D}\right)^{1 / 2} \sim \Lambda_{Y M}=\left(\Lambda_{Q}^{\mathrm{b}_{\mathrm{o}}} m_{Q}^{N_{F}}\right)^{1 / 3 N_{c}} \ll \Lambda_{\mathrm{ch}}^{S Q C D} \sim \mathcal{M}_{\mathrm{ch}} \ll \Lambda_{Q}$.

c) So, the heavy quarkonia (meson and baryon) masses are also parametrically different: $M_{\text {meson }}^{Q C D} \sim\left(\Lambda_{\text {ch }}^{Q C D}+\Lambda_{\text {conf }}^{Q C D}\right) \sim \Lambda_{Q}, M_{\text {baryon }}^{Q C D} \sim N_{c} \Lambda_{\text {ch }}^{Q C D} \sim N_{c} \Lambda_{Q}$ in QCD, while they are $M_{\text {meson }}^{S Q C D} \sim \mathcal{M}_{\mathrm{ch}} \ll \Lambda_{Q}, M_{\text {baryon }}^{S Q C D} \sim N_{c} \mathcal{M}_{\text {ch }}$ in SQCD.

d) The masses of gluonia are $M_{\mathrm{gl}}^{Q C D} \sim \Lambda_{\text {conf }}^{Q C D} \sim \Lambda_{Q}$ in QCD, while they are $M_{\mathrm{gl}}^{S Q C D} \sim$ $\Lambda_{\text {conf }}^{S Q C D} \sim \Lambda_{Y M} \ll \mathcal{M}_{\text {ch }} \ll \Lambda_{Q}$ in SQCD.

e) The smallest pion masses are $M_{\pi}^{Q C D} \sim\left(m_{Q} \Lambda_{\mathrm{ch}}^{Q C D}\right)^{1 / 2} \sim\left(m_{Q} \Lambda_{Q}\right)^{1 / 2} \gg m_{Q}$ in QCD, while they are not $\sim\left(m_{Q} \mathcal{M}_{\mathrm{ch}}\right)^{1 / 2}$, but $M_{\pi}^{S Q C D} \sim m_{Q}$ in SQCD (this last difference is because the spin 1/2 quarks are condensed in QCD, while these are spin zero quarks in SQCD).

Now, let us comment briefly on the $N_{c}$-dependence of various quantities that appeared in the text above. The standard $N_{c}$-counting rules predict that the gluino and quark condensates, $\langle S\rangle$ and $\left\langle\bar{Q}^{\bar{j}} Q_{i}\right\rangle$, are not $O(1)$ at $N_{c} \gg 1, N_{F} / N_{c}=$ const, as in the text, but $N_{c}$ times larger, $O\left(N_{c}\right)$ (and this agrees with explicit calculations, see e.g. [11]). 21 The right dependence on $N_{c}$ can easily be restored over all the text by simple substitutions, for instance, $\Lambda_{Q}^{\mathrm{b}_{\mathrm{o}}} \rightarrow N_{c}^{N_{c}} \Lambda_{Q}^{\mathrm{b}_{\mathrm{o}}}$ in $(3.13)$, etc.

20 QCD means here our QCD with $N_{c}=3$ and $N_{F} \simeq 3$ of light flavors.

21 Besides, this can be seen from the example with $N_{F}<N_{c}$, when quarks are higgsed (see section $2)$. The gluon masses, $\mu_{\mathrm{gl}}^{2} \sim \alpha\left(\mu=\mu_{\mathrm{gl}}\right) \mathcal{M}_{o}^{2}$, are $O(1)$. Because $\alpha=O\left(1 / N_{c}\right), \mathcal{M}_{o}^{2}$ is $O\left(N_{c}\right),\langle S\rangle=$ $\hat{m}_{Q} \mathcal{M}_{o}^{2}=O\left(N_{c}\right)$.

Connected with this, there is the inherent ambiguity in the VY-procedure for the pure Yang-Mills theory: one can replace $\ln \left(\mu^{3} / \Lambda^{3}\right)$ by $\ln \left(S / C_{o} \Lambda^{3}\right)-1$, where $C_{o}$ is some constant. The value $C_{o}=1$ was used everywhere in the text, while $\mu^{3}$ is definitely $N_{c}$-independent, so that a better replacement is rather $: \ln \left(\mu^{3} / \Lambda^{3}\right) \rightarrow \ln \left(S / N_{c} \Lambda^{3}\right)-1$, resulting in $\langle S\rangle=N_{c} \Lambda^{3}$. 
Finally, we would like to make a comment about the spontaneously SUSY-breaking metastable local vacuum in SQCD with $N_{c}+1<N_{F}<3 N_{c} / 2, m_{Q} \neq 0, m_{Q} \ll \Lambda_{Q}$, proposed recently in [12. The arguments for the existence of such a state in the dual theory are presented in [12].

Recalling general arguments given above in section 7 (see (7.2)-(7.4), it is worth also recalling that these arguments are not connected with the use of the dynamical scenario with the diquark-condensate) that the direct and dual theories are not equivalent in the infrared region, it becomes insufficient to show such a state in the dual theory, because this does not imply automatically that this state exists also in the direct theory. So, let us try to find out this state within the direct theory.

In terms of the direct theory fields, this state is characterized by all $N_{F}^{2}$ components $\left\langle M \frac{i}{j}\right\rangle=\left\langle\bar{Q}{ }_{\bar{j}} Q^{i}\right\rangle=0$, while $\langle B\rangle=\operatorname{const}\langle b\rangle \neq 0$ (and $\langle\bar{B}\rangle$ the same), $B \rightarrow Q^{N_{c}}, b \rightarrow q^{\bar{N}_{c}}$. Unfortunately, no simple possibility for a local vacuum with these properties is seen in the direct theory. For instance, the dynamics underlying the appearance of the above basic nonzero baryon condensates looks obscure. If these baryon condensates were, for instance, due to higgsed quarks $\left\langle Q^{i}\right\rangle=\left\langle\bar{Q}_{\bar{j}}\right\rangle \neq 0$, with $i, \bar{j}=1 \ldots N_{c}$, so that $\langle B\rangle=$ $\langle\bar{B}\rangle \sim\left\langle Q^{i}\right\rangle^{N_{c}} \neq 0$, then no reason is seen for all components of $\left\langle M_{\bar{j}}^{i}\right\rangle=\left\langle\bar{Q}_{\bar{j}} Q^{i}\right\rangle$ to be exactly zero. Rather, $\left\langle M \frac{i}{j}\right\rangle$ with $i=\bar{j}=1 \ldots N_{c}$ will be $\sim\left\langle Q^{i}\right\rangle\left\langle\bar{Q}_{i}\right\rangle \neq 0$. Besides, looking at the Lagrangian in (3.2) it is seen that it becomes singular at $\mathcal{M}_{\mathrm{ch}} \rightarrow 0$. So, it seems impossible that the local vacuum with the above given properties can appear here.

However, this is not the whole story as (3.2) is a local Lagrangian, i.e. it is valid only locally in the field space, not too far from the genuine SUSY-vacuum. This implies that generally, besides $M_{\bar{j}}^{i}$, the additional fields can be involved to describe correctly the vicinity of the above metastable vacuum. So, let us try in addition from another side, using some specific properties of the above metastable state of the dual theory. Let us also look at the lightest excitations around this vacuum. As was argued in [12], all excitations have masses $\sim\left(m_{Q} \Lambda_{Q}\right)^{1 / 2}$, except for some massless modes of the baryon and $M_{\bar{j}}^{i}=\left(\bar{Q} \bar{j}^{i}\right)$ fields (and the basic vacuum condensates of baryons). So, let us take the scale $\mu \ll\left(m_{Q} \Lambda_{Q}\right)^{1 / 2}$ and try to write by hand the effective superpotential made of these meson and baryon fields only. The simplest form is:

$$
W_{\text {eff }}=-\bar{N}_{c}\left\{\frac{\operatorname{det} \mathrm{M}-\operatorname{Tr}\left(\overline{\mathrm{B}} \mathrm{M}^{\left.\overline{\mathrm{N}}_{\mathrm{c}} \mathrm{B}\right)}\right.}{\Lambda_{\mathrm{Q}}^{\mathrm{b}_{\mathrm{o}}}}\right\}^{1 / \overline{\mathrm{N}}_{\mathrm{c}}}+\mathrm{m}_{\mathrm{Q}} \operatorname{Tr} \mathrm{M} .
$$

At $\bar{N}_{c} \geq 2$ no possibility is seen to obtain from (9.1) the non-singular expansion in quantum fluctuations around the state with $\langle M\rangle=0,\langle B\rangle=\langle\bar{B}\rangle \neq 0$. 22 Only the case $\bar{N}_{c}=1$ is non-singular in (9.1). But even in this case one has to show then how it is possible to obtain in some way (9.1) starting with (2.1) and expanding self-consistently around this metastable vacuum. It seems, there will be problems.

Finally, the absence of the above metastable spontaneously SUSY-breaking state in the direct theory may be not so surprising, taking into account all arguments given above in the text that the direct and dual theories are not equivalent.

This work is supported in part by the RFBR grant 07-02-00361-a.

\footnotetext{
22 Formally, one can multiply the first term in the r.h.s. of (9.1) by a function $f(z), z=$ $\operatorname{det} \mathrm{M} / \operatorname{Tr}\left(\overline{\mathrm{B}} \mathrm{M}^{\overline{\mathrm{N}}_{\mathrm{c}}} \mathrm{B}\right)$, but this does not help to avoid singularities.
} 


\section{Appendix}

The purpose of this appendix is to comment in short on a situation with anomalous divergences of external currents (the 't Hooft triangles) in SQCD, within the dynamical scenario considered in this paper.

In our ordinary QCD, at the scale $\mu_{\mathrm{ch}} \sim \Lambda_{Q}$ and at $m_{Q} \rightarrow 0$, there is the genuine spontaneous breaking of the flavor symmetry: $S U\left(N_{F}\right)_{L} \times S U\left(N_{F}\right)_{R} \rightarrow S U\left(N_{F}\right)_{L+R}$, while the baryon symmetry $U(1)_{B}$ remains unbroken. So, the quarks acquire the constituent masses $\mu_{C} \sim \mu_{\mathrm{ch}}$ and decouple at $\mu<\mu_{\mathrm{ch}}$ (together with all gluons which acquire (either electric or magnetic) masses $\sim \Lambda_{Q}$ due to non-perturbative confining interactions, so that the lower energy theory contains only $\left(N_{F}^{2}-1\right)$ light pions. If the quarks are exactly massless, the pions are also massless, while if the chiral symmetry $S U\left(N_{F}\right)_{L} \times S U\left(N_{F}\right)_{R}$ is broken explicitly down to $S U\left(N_{F}\right)_{L+R}$ by parametrically small quark masses $0<m_{Q} \ll \Lambda_{Q}$, the pions become the pseudo-Goldstone bosons with parametrically small masses $m_{\pi} \sim\left(m_{Q} \Lambda_{Q}\right)^{1 / 2} \ll \mu_{\mathrm{ch}}$.

In SQCD with $N_{F}<N_{c}$ and with small explicit breaking of chiral flavor symmetry and R-charge by quark masses $0<m_{Q} \ll \Lambda_{Q}$ (see section 2), the scalar quarks are higgsed at the high scale $\mu_{\mathrm{ch}}=\mu_{\mathrm{gl}} \gg \Lambda_{Q}\left(\mu_{\mathrm{gl}} \simeq \mathcal{M}_{\mathrm{ch}}\right.$, with the logarithmic accuracy) and acquire the large "constituent masses" $\mu_{C}=\mu_{\mathrm{gl}}$. The color symmetry $S U\left(N_{c}\right)$ is broken down to $S U\left(N_{c}-N_{F}\right)$, and $\left(2 N_{c} N_{F}-N_{F}^{2}\right)$ gluons become massive eating the Goldstone bosons. So, all this can be considered as the quasi-spontaneous symmetry breaking: $S U\left(N_{c}\right)_{C} \times$ $S U\left(N_{F}\right)_{L} \times S U\left(N_{F}\right)_{R} \times U(1)_{R} \times U(1)_{B} \rightarrow S U\left(N_{c}-N_{F}\right)_{C} \times S U\left(N_{F}\right)_{C+L+R} \times U(1)_{B}$, as the "constituent masses" $\mu_{C} \sim \mathcal{M}_{\mathrm{ch}}$ are parametrically larger than the pion masses $m_{\pi} \sim m_{Q}$ (with the logarithmic accuracy). As a result, there appear $N_{F}^{2}$ pseudo-Goldstone pions (together with their superpartners). So, the lower energy theory at $\mu<\mu_{\mathrm{gl}}$ includes the superfields of light $\left(N_{c}-N_{F}\right)^{2}-1$ gluons and $N_{F}^{2}$ pions.

In SQCD with $N_{F}>N_{c}$ and $m_{Q} \ll \Lambda_{Q}$ (in the dynamical scenario considered in this paper), all quarks acquire the constituent masses $\mu_{C}=\mathcal{M}_{\text {ch }} \ll \Lambda_{Q}$ in the threshold region $\mu \sim \mu_{\mathrm{ch}}=\mathcal{M}_{\mathrm{ch}}$, and there appear $N_{F}^{2}$ light pions, while all gluons remain massless. This also can be considered as the quasi-spontaneous symmetry breaking: $S U\left(N_{F}\right)_{L} \times S U\left(N_{F}\right)_{R} \times U(1)_{R} \times U(1)_{B} \rightarrow S U\left(N_{F}\right)_{L+R} \times U(1)_{B}$, as the constituent quark masses $\mu_{C}$ are parametrically larger than the pion masses $m_{\pi} \sim m_{Q} \ll \mathcal{M}_{\text {ch }}$. The lower energy theory at $\mu<\mathcal{M}_{\mathrm{ch}}$ includes the superfields of light $\left(N_{c}^{2}-1\right)$ gluons and $N_{F}^{2}$ pions.

Let us recall now some important and well known properties of the lower energy theory at $\mu<\mu_{\mathrm{ch}}$.

1) After integrating out all heavy fields (and all Fourier components of light fields with $k>\mu_{\mathrm{ch}}$ ), the Lagrangian of the lower energy theory at $\mu<\mu_{\mathrm{ch}}$ will be local, right because all integrated modes were hard (it is always implied that this integration is performed in a way which respects all symmetries).

2) The external global symmetries can be gauged by introducing external vector fields and adding the appropriate set of massless "leptons", so that all anomalous divergences of external currents originating from the quark-gluon sector will be canceled by those originating from the lepton one.

3) After all this, because the symmetry breaking in the quark-gluon sector was quasispontaneous, the lower energy Lagrangian will preserve all previous symmetries, both internal and external. So, because nothing happens with leptons when crossing the scale 
$\mu=\mu_{\mathrm{ch}}$, the anomalous divergences originating from the quark-gluon sector also remain the same [13].

So, there is no questions whether the lower energy theory behaves properly under symmetry transformations, both internal and external, or whether the anomalous divergences of external currents originating from the quark-gluon sector will remain the same in the lower energy theory, ${ }^{23}$ as they were in the higher energy theory at $\mu>\mu_{\mathrm{ch}}$, - this is automatic. The only relevant questions are: a) what is the explicit form of the lower energy Lagrangian; b) in what way, explicitly, the anomalous divergences of external currents originating from the quark-gluon sector are saturated by fields of the lower energy theory.

As for 'a', if the dynamics of the theory is under a full control, the explicit form of the lower energy Lagrangian is obtained by the above described direct integration. As is well known, besides the 'standard terms', there will appear additional Wess-Zumino-like terms [14] 15$]$.

Now, a few words about 'b', within the dynamical scenario for SQCD considered in this paper. First, as for pions, it is worth noting that because contributions of pion loops are power suppressed at scales $\mu<\mu_{\mathrm{ch}}$, these loops will give only small power corrections to the contributions of tree diagrams into amplitudes with low energy external pions and/or external gauge fields.

There will appear one-pion terms, $J_{\nu}^{\text {ext }} \sim i F_{\pi} \partial_{\nu} \pi+\ldots$, in those external currents which correspond to quasi-spontaneously broken generators, with the pion decay constant $F_{\pi} \sim \mathcal{M}_{o}$ for $N_{F}<N_{c}$, and $F_{\pi} \sim \mathcal{M}_{\mathrm{ch}}$ for $N_{c}<N_{F}<3 N_{c}$. Besides, among many others, there will be the well known term $\sim F_{\pi}^{-1} \operatorname{Tr}\left(\pi F_{\mu \nu} \tilde{F}_{\mu \nu}\right)$ in the Wess-Zumino part of the Lagrangian (here $F_{\mu \nu}$ is the field strength of the external vector fields, $W_{L}$ or $W_{R}$ - bosons, or R-photon $A^{R}$ ), with the appropriate coefficient. As a result, the anomalous divergences of all such currents will be automatically saturated by a sum of three contributions: a) the one intermediate pion exchange, b) the direct contributions into triangles of fermionic pion superpartners, c) the additional direct contributions of gluinos into $R$ and $R^{3}$ triangles.

So, for instance, for all $N_{c}<N_{F}<3 N_{c}$ (with the logarithmic accuracy for $N_{F}<N_{c}$ ), the decay width of the pion into two (sufficiently light at small $\alpha_{\text {ext }}$ ) vector bosons $V=$ $\left\{W_{L}, W_{R}, A^{R}\right\}$ will be: $\Gamma(\pi \rightarrow 2 V) \sim \alpha_{\text {ext }}^{2} m_{\pi}^{3} / F_{\pi}^{2} \sim \alpha_{\text {ext }}^{2} m_{Q}^{3} / \mathcal{M}_{\text {ch }}^{2} \sim \alpha_{\text {ext }}^{2} \Lambda_{Q}\left(m_{Q} / \Lambda_{Q}\right)^{\Delta}, \Delta=$ $\left(4 N_{c}-N_{F}\right) / N_{c}$.

Those external currents, e.g. the baryon one, which corresponds to the unbroken generators, will not contain the one-pion term (because there is no corresponding pion), and their anomalous divergences, like $\left\langle W_{L}\left|\partial_{\nu} J_{\nu}^{B}\right| W_{L}\right\rangle$, will be directly saturated by the point-like terms $\sim\left(\epsilon_{\nu \lambda \sigma \tau} A_{\nu}^{B} W_{\lambda}^{L} \partial_{\sigma} W_{\tau}^{L}+\ldots\right)$ in the Wess-Zumino part of the Lagrangian.

We did not write explicitly in the main text the Wess-Zumino-like terms because: a) this is not a simple matter to find their explicit form, b) they are irrelevant for the main purpose of this paper - to calculate the mass spectrum of the theory.

23 i.e. at scales $\mu_{\text {expl }}<\mu<\mu_{\text {ch }}$, where $\mu_{\text {expl }} \sim m_{\pi} \ll \mu_{\text {ch }}$ is the scale of the explicit global chiral symmetry breaking, because the explicitly broken global symmetry is incompatible with gauging this symmetry, and $\mu_{\text {expl }}$ can be neglected only at scales $\mu>\mu_{\text {expl }}$. 


\section{References}

[1] N. Seiberg, Phys. Rev. D 49 (1994) 6857, hep-th/9402044

N. Seiberg, Nucl. Phys. B435 (1995) 129, hep-th/9411149

[2] D. Amati, K. Konishi, Y. Meurice, G.C. Rossi, G. Veneziano, Phys. Rep. 162 (1988) 169, and references therein

[3] K. Intriligator, N. Seiberg, Nucl. Phys. Proc. Suppl., 45BC (1996) 1, hep-th/9509066

[4] M. Shifman, Prog. Part. Nucl. Phys. 39 (1997) 1, hep-th/9704114

[5] V. Novikov, M. Shifman, A. Vainshtein, V. Zakharov, Nucl. Phys. B229 (1983) 381, Phys. Lett. B166 (1986) 329;

M. Shifman, A. Vainshtein, Nucl. Phys. B277 (1986) 456

[6] G. Veneziano, S. Yankielowicz, Phys. Lett. B 113 (1982) 321

T. Taylor, G. Veneziano, S. Yankielowicz, Nuc. Phys. B218 (1983) 493

[7] K. Konishi, Phys. Lett. B135 (1984) 439

[8] I. Affleck, M. Dine, N. Seiberg, Nucl. Phys. B241 (1984) 493;

B256 (1985) 557

[9] G.R. Dvali, M.A. Shifman, Phys. Lett. B396 (1997) 64, hep-th/9612128

[10] I. Kogan, M. Shifman, A. Vainshtein, Phys. Rev. D53 (1996) 4526, Err: D59 (1999) 109903; hep-th/9507170

[11] M. Shifman, A. Vainshtein, hep-th/9902018 and references therein

[12] K. Intriligator, N. Seiberg, D. Shih, JHEP, 0604 (2006) 021, hep-th/0602239

K. Intriligator, N. Seiberg, hep-ph/0702069

[13] G. 't Hooft, Recent Developments in Gauge Theories, Eds. G. 't Hooft et al., Plenum Press, New York, 1980

[14] J. Wess, B. Zumino, Phys. Lett. B37 (1971) 95

[15] J. Goldstone, F. Wilczek, Phys. Rev. Lett. 47 (1981) 986

E. Witten, Nucl. Phys. B223 (1983) 422 